\title{
Finite-Larmor-radius equilibrium and currents of the Earth's flank magnetopause
}

\author{
S. S. Cerri $\dagger$ \\ Department of Astrophysical Sciences, Princeton University, Princeton, NJ 08544, USA
}

(Received 5 April 2018; revised 14 August 2018; accepted 15 August 2018)

We consider the one-dimensional equilibrium problem of a shear-flow boundary layer within an 'extended-fluid model' of a plasma that includes the Hall and the electron pressure terms in Ohm's law, as well as dynamic equations for anisotropic pressure for each species and first-order finite-Larmor-radius (FLR) corrections to the ion dynamics. We provide a generalized version of the analytic expressions for the equilibrium configuration given in Cerri et al., (Phys. Plasmas, vol. 20 (11), 2013, 112112), highlighting their intrinsic asymmetry due to the relative orientation of the magnetic field $\boldsymbol{B}, \boldsymbol{b}=\boldsymbol{B} /|\boldsymbol{B}|$, and the fluid vorticity $\boldsymbol{\omega}=\boldsymbol{\nabla} \times \boldsymbol{u}$ (' $\boldsymbol{\omega} \boldsymbol{b}$ asymmetry'). Finally, we show that FLR effects can modify the Chapman-Ferraro current layer at the flank magnetopause in a way that is consistent with the observed structure reported by Haaland et al., (J. Geophys. Res. (Space Phys.), vol. 119, 2014, pp. 9019-9037). In particular, we are able to qualitatively reproduce the following key features: (i) the dusk-dawn asymmetry of the current layer, (ii) a double-peak feature in the current profiles and (iii) adjacent current sheets having thicknesses of several ion Larmor radii and with different current directions.

Key words: space plasma physics, plasma flows

\section{Introduction}

A comprehensive modelling of magnetized plasmas and of their multi-scale dynamics is an outstanding challenge in laboratory, astrophysical and space plasma research. In particular, given that direct numerical simulations are nowadays the main tool to address such complex dynamics, finding a compromise between an exhaustive theoretical model and its actual implementation represents a major goal for computational plasma physics.

A kinetic model based on the full Vlasov-Maxwell system of equations would need to be solved in a six-dimensional phase space (three real-space and three velocity-space dimensions), resolving length and time scales that typically span over several orders of magnitude. For this reason, fully kinetic simulations that adopt realistic parameters and/or complex geometries are still far from being realizable because of their colossal computational cost. Moreover, there is overwhelming difficulty in constructing an analytical description of Vlasov equilibria in realistic settings. In fact, the few existing examples typically consider very simplified cases

\footnotetext{
$\dagger$ Email address for correspondence: scerri@astro.princeton.edu
} 
(e.g. uniform and homogeneous magnetic field and/or only periodic functions) and still one cannot fully constrain the resulting velocity profiles beforehand and/or provide those equilibria without appealing to a numerical solution of the problem (see, e.g. Cai, Storey \& Neubert 1990; Attico \& Pegoraro 1999; Mahajan \& Hazeltine 2000; Bobrova et al. 2001; Malara, Pezzi \& Valentini 2018).

On the other hand, a model based on a fluid treatment such as the magnetohydrodynamic (MHD) equations neglects most of the characteristic length and time scales inherent to a kinetic description of the plasma dynamics and only needs to be solved in real space. The MHD description thus represents the simplest viable approach, which nevertheless has led to many fundamental theoretical results (e.g. Chapman \& Ferraro 1930; Ferraro 1937; Alfvén 1942; Lüst \& Schlüter 1954; Chandrasekhar 1956; Shafranov 1958; Grad 1960; Taylor 1974). Furthermore, in the last two decades, we have been able to afford well-resolved MHD global simulations providing useful insights (e.g. Groth et al. 2000; Siscoe et al. 2000; Jia et al. 2012, 2015; Merkin, Lyon \& Claudepierre 2013; Liu et al. 2015; Dong et al. 2017; Sorathia et al. 2017). However, in a real system, the nonlinear plasma dynamics would naturally develop small scales and bring the effects associated with the neglected kinetic scales back to light, and so a MHD description eventually breaks down. Moreover, accounting for the leading kinetic effects may already be necessary to implement a correct initial plasma equilibrium, in order to avoid uncontrolled and spurious readjustments that can affect the subsequent dynamics or to explain certain features of the system under consideration (e.g. Cerri et al. 2013; Henri et al. 2013).

The fully kinetic and MHD descriptions actually represent the two extremes of a wide variety of plasma models. There are a large number of approaches that try to bridge the above antipodes in different ways: from the one side, by simplifying a fully kinetic description based on the dismissal of presumably unimportant effects; from the opposite side, by gradually including more and more kinetic effects within a fluid framework. The former class of models are usually referred to as 'reducedkinetic models', such as the gyrokinetic (GK) (Brizard \& Hahm 2007) and the hybrid Vlasov-Maxwell (HVM) (Valentini et al. 2007) approximations; the latter are known as 'extended-fluid models', in which kinetic effects are gradually included in a fluid description. This is the case, for instance, when retaining finite-Larmor-radius (FLR) corrections (Roberts \& Taylor 1962; Macmahon 1965), or when including the effect of linear Landau damping (Landau 1946) by modelling it with a so-called Landaufluid (LF) closure (e.g. Hammett \& Perkins 1990). These two aspects can also be both included within a single framework, such as in the so-called finite-Larmor-radius Landau-fluid (FLRLF) model (Sulem \& Passot 2015). However, within the range of validity defined by each model's assumption ('ordering'), reduced-kinetic models still unavoidably face the curse of high dimensionality, and so extended-fluid models still represent an attractive choice when seeking a compromise between kinetic and fluid descriptions.

The need to extend a standard fluid description of a collisionless plasma to include at least these effects related to a non-gyrotropic pressure tensor is particularly evident when a sheared flow is present: in the collisionless regime, due to FLR effects, the pressure tensor is indeed strongly coupled to the shear flow and they interact over very short time scales (Cerri 2012; Cerri et al. 2013, 2014; Del Sarto, Pegoraro \& Califano 2016; Del Sarto, Pegoraro \& Tenerani 2017; Del Sarto \& Pegoraro 2018). This is exactly the case of the low-latitude boundary layer (LLBL) between the solar-wind flow and the Earth's magnetosphere, where the velocity shear drives the Kelvin-Helmholtz instability (KHI) that generates the observed large-scale 'MHD' 
vortices (see, e.g. Faganello \& Califano 2017 and references therein). In such a region, in addition to the vortex dynamics that naturally develops fluctuations on length scales comparable to (or even smaller than) the ion gyroradius $\varrho_{i}$ (or the ion inertial length $d_{i}$ ), the 'large-scale' equilibrium fields and the sheared flow itself vary over typical length scales $L_{0}$ that do not exceed the ion characteristic scales by a large amount, and so ' $\varrho_{i} / L_{0}$ corrections' cannot be completely neglected. So far, such a system has been modelled by means of one-dimensional isotropic MHD equilibrium configurations that ensure the total pressure balance, i.e. a balance between the thermal and magnetic scalar pressures of the two plasmas without involving the properties of the background sheared flow. However, as soon as FLR effects and/or the full ion pressure tensor are taken into account, the shear-flow properties enter the pressure-balance conditions and the simple isotropic MHD configurations are generally no longer an equilibrium (Cerri 2012; Cerri et al. 2013, 2014). As a result, the system naturally develops shear-driven anisotropies (e.g. De Camillis et al. 2016; Del Sarto et al. 2016; Del Sarto \& Pegoraro 2018). This is important for (at least) two practical reasons. First, a difficulty arises when comparing the linear evolution of the KHI using fluid and kinetic models. As discussed in Henri et al. (2013), in which the same isotropic MHD configuration was adopted as an initial condition for simulations using different plasma models (namely, MHD, two-fluid, hybrid and full Particle-In-Cell (PIC)), it was found that violent and uncontrolled readjustments were injecting large-amplitude fluctuations into the system (see also Del Sarto et al. 2017) and changing the configuration on top of which the instability develops (see also Nakamura, Hasegawa \& Shinohara 2010). Therefore, these spurious effects would partially mask the actual kinetic effects on the KHI and make a genuine comparison difficult. Secondly, using ten years of observations made by the Cluster satellites, Haaland et al. (2014) have recently highlighted that the Earth's magnetopause exhibits a current structure that is more complex than the simple MHD layer described by Chapman \& Ferraro (1930), as well as a clear asymmetry between the dusk and the dawn sides. In addition to the implications for the current system of a planet magnetosphere, these ion-kinetic effects can indeed cause the asymmetric development of KHI at the dawn and the dusk sides of such magnetosphere, as well as other non-ideal effects (e.g. Nagano 1978; Huba 1996; Terada, Machida \& Shinagawa 2002; Nakamura et al. 2010; Henri et al. 2012; Masters et al. 2012; Sundberg et al. 2012; Taylor et al. 2012; Delamere et al. 2013; Paral \& Rankin 2013; Haaland et al. 2014; Johnson, Wing \& Delamere 2014; Liljeblad et al. 2014; Walsh et al. 2014; Gershman et al. 2015; Gingell, Sundberg \& Burgess 2015; De Camillis et al. 2016).

The aim of the present work is to show how the non-ideal behaviour of the Chapman-Ferraro layer could be qualitatively understood in terms of a onedimensional equilibrium of the shear-flow layer within an extended-fluid model that includes first-order ion-FLR corrections. The great simplicity of the treatment presented here allows us to derive analytical equilibrium profiles in which the ion-kinetic effects can be clearly identified. Therefore this study is meant to be a first step - a sort of 'proof of concept' - towards the identification of the effects possibly leading to the observed behaviour of the low-latitude magnetopause layer, rather than an exhaustive description of the actual system. In order to achieve a quantitative modelling of the global magnetopause current system within this (or a more comprehensive) extended-fluid model, a numerical approach to the solution of the full three-dimensional problem would likely be required.

The remainder of this paper is organized as follows. In $\S 2$ we describe the extended two-fluid (eTF) model of Cerri et al. (2013) and we outline the procedure 
for the derivation of the equilibrium profiles (the actual derivation of a general family of solutions for the shear-flow boundary layer equilibrium is provided in appendix A); consequences for shear-flow instabilities, agyrotropy and links to turbulent environments are highlighted in $\$ \S 2.3-2.5$. In $\S 3$ we show how these profiles can qualitatively explain the observed non-ideal behaviour of the LLBL between the solar wind and the Earth's magnetosphere. Finally, in $\S 4$ conclusions are drawn. Additionally, explicit considerations on the symmetries of the FLR expansion and on its convergence to a full pressure tensor case are reported in appendices B and $\mathrm{C}$, respectively.

\section{The extended two-fluid (eTF) model}

Here, we consider a non-relativistic quasi-neutral proton-electron plasma $\left(n_{\mathrm{p}} \simeq n_{\mathrm{e}} \equiv\right.$ $n)$ in the limit of massless electrons, $m_{\mathrm{e}} / m_{\mathrm{p}} \rightarrow 0$. The model includes the Hall and the electron pressure terms in the generalized Ohm's law, as well as dynamic equations for the gyrotropic pressures of both species and first-order FLR corrections to the protons' pressure tensor ${ }^{1}$. The fluid hierarchy is closed with a double-adiabatic approximation, i.e. by neglecting the heat fluxes, $q_{\|}=0$ and $q_{\perp}=0$. Such assumption is indeed justified within a finite-but-small Larmor-radius expansion and on time scales much longer than the ion cyclotron time scale, $\rho / L \sim \omega / \Omega \sim \varepsilon \ll 1$, where $\rho$ is the thermal Larmor radius, $L$ is the typical length scale of variation for macroscopic quantities and $\Omega$ is the cyclotron frequency (see Cerri et al. 2013, for explicit equations and further details about the eTF model ordering). In fact, by neglecting gradients in the direction of the magnetic field $\left(\boldsymbol{b} \cdot \nabla=\nabla_{\|}=0\right.$; see appendix A), the expressions for the perpendicular heat fluxes (see, e.g. Braginskii 1965; Ramos 2008) would give a second-order contribution which is ordered out in the eTF model ${ }^{2}$. In this model, the thermal pressure tensor of the protons and of the electrons, $\boldsymbol{\Pi}_{\mathrm{p}}$ and $\boldsymbol{\Pi}_{\mathrm{e}}$ respectively, are written as

$$
\begin{gathered}
\boldsymbol{\Pi}_{\mathrm{p}}=p_{\| \mathrm{p}} \boldsymbol{b} \boldsymbol{b}+p_{\perp \mathrm{p}} \boldsymbol{\tau}+\pi_{\mathrm{p}}^{(1)} \\
\boldsymbol{\Pi}_{\mathrm{e}}=p_{\| \mathrm{e}} \boldsymbol{b} \boldsymbol{b}+p_{\perp \mathrm{e}} \boldsymbol{\tau}
\end{gathered}
$$

where $\boldsymbol{b} \equiv \boldsymbol{B} /|\boldsymbol{B}|$ is the magnetic-field unit vector, $\boldsymbol{\tau} \equiv \boldsymbol{I}-\boldsymbol{b} \boldsymbol{b}$ is the projector onto the plane perpendicular to $\boldsymbol{B}$ and $p_{\| \alpha}$ and $p_{\perp \alpha}$ are the gyrotropic thermal pressures of the $\alpha$ species parallel and perpendicular to the magnetic field, respectively (Chew, Goldberger \& Low 1956). In (2.1), $\pi_{\mathrm{p}}^{(1)}$ is a traceless symmetric tensor taking into account first-order FLR corrections to the gyrotropic proton pressure (also known as gyroviscous tensor). Neglecting the heat fluxes, a general formulation for the

\footnotetext{
${ }^{1}$ We note that in the existing literature the name 'extended MHD' is sometimes used to describe magnetohydrodynamic models that include Hall terms and electron inertia effects (see, e.g. Kimura \& Morrison 2014). Hereafter, we will instead refer to a model as an 'extended fluid model' when certain kinetic effects, such as, for instance, finite-Larmor-radius contributions and/or linear models of Landau damping, are included within a fluid description.

${ }^{2}$ This can be seen also from the point of view of the time scales involved. Let us consider the expressions for the heat fluxes given in Ramos (2008), that in the configuration considered here will reduce to $\boldsymbol{q}_{\perp}=$ $\left(2 p_{\perp} / m \Omega\right) \boldsymbol{b} \times \nabla T_{\perp}$ and $\boldsymbol{q}_{\|}=\left(p_{\perp} / 2 m \Omega\right) \boldsymbol{b} \times \nabla T_{\|}$. The time scale on which the divergence of these heat fluxes would contribute on the pressure evolution is thus $\tau_{\nabla q} \sim\left(L_{\perp} / \rho\right)^{2} \Omega^{-1} \sim \varepsilon^{-2} \Omega^{-1}$ (the time scale for $q_{\|}$would actually involve an additional anisotropy correction, $T_{\|} / T_{\perp}$, which is not very relevant here). Therefore, the divergence of the heat flux can be neglected with respect to the flow time scale as long as $\varepsilon \sqrt{\beta_{\perp}} \ll u / v_{A}(u$ is the typical flow velocity and $v_{A}$ is the Alfvén speed), which is satisfied for the cases under study.
} 
gyroviscous tensor components can be written as (Macmahon 1965; Schekochihin et al. 2010; Sulem \& Passot 2015)

$$
\pi_{\mathrm{p}, i j}^{(1)}=\frac{p_{\perp \mathrm{p}}}{4 \Omega_{c \mathrm{p}}}\left(\epsilon_{i l m} b_{l} S_{m k} H_{k j}-H_{i k} \epsilon_{j l m} S_{k l} b_{m}\right)+\frac{2\left(p_{\perp \mathrm{p}}-p_{\| \mathrm{p}}\right)}{\Omega_{c \mathrm{p}}}\left(b_{i} w_{j}+b_{j} w_{i}\right),
$$

where $\Omega_{c \mathrm{p}}=e B / m_{\mathrm{p}} c$ is the proton gyro-frequency, $\epsilon_{i j k}$ is the completely antisymmetric Levi-Civita tensor, and we have introduced $S_{i j} \equiv \partial_{i} u_{\mathrm{p}, j}+\partial_{j} u_{\mathrm{p}, i}, H_{i j} \equiv \delta_{i j}+3 b_{i} b_{j}$ and $w_{i} \equiv \epsilon_{i j k}\left(\nabla_{\|} u_{\mathrm{p}, j}\right) b_{k}$, with $\nabla_{\|} \equiv \boldsymbol{b} \cdot \nabla$. Note that the above formulation automatically takes into account for the asymmetry due to the magnetic field direction with respect to the vorticity (see also appendix B and Cerri et al. 2013, for explicit symmetry considerations).

\subsection{Shear-flow layer equilibrium with FLR}

Within this model, we now outline the derivation of equilibrium profiles for a one-dimensional velocity-shear layer separating, for instance, two different plasmas. The explicit derivation of this class of analytical solutions that generalize the results provided in Cerri et al. (2013) and that include a much wider range of configurations of interest for what concerns magnetospheric observations will be provided in appendix A. The goal is to provide an equilibrium configuration with FLR corrections for the flank magnetopause, and to discuss the implications on the low-latitude boundary layer (LLBL) profiles. For the sake of simplicity, here we consider the one-dimensional equilibrium problem, which can be seen as a local approximation of the LLBL. A global treatment of the magnetospheric structure should take into account curvature terms, as well as possible gradients parallel to the magnetic field and compressible flows. This may need to include additional equilibrium conditions that involve all the gyroviscous components and eventually to go beyond the simple adiabatic FLR treatment presented here by, for instance, including heat fluxes (see, e.g. Sulem \& Passot 2015; Del Sarto \& Pegoraro 2018).

We consider a given $x$-dependent incompressible MHD flow in the $y-z$ plane,

$$
\boldsymbol{u}=u_{y}(x) \boldsymbol{e}_{y}+u_{z}(x) \boldsymbol{e}_{z}, \quad \nabla \cdot \boldsymbol{u}=0,
$$

such that it becomes constant at the boundaries (i.e. we consider a localized velocityshear layer). The magnetic field also lies on the $y-z$ plane,

$$
\boldsymbol{B}(x)=B_{y}(x) \boldsymbol{e}_{y}+B_{z}(x) \boldsymbol{e}_{z} .
$$

We further simplify the problem by assuming a polytropic relation for the thermal pressures $^{3}$. This assumption is not strictly necessary in order to derive the equilibrium, but it is useful for providing density and temperature profiles from the obtained pressure profiles. In general, the equilibrium for this configuration is found by imposing total pressure balance:

$$
\frac{\mathrm{d}}{\mathrm{d} x}\left[\boldsymbol{\Pi}_{\mathrm{p}}(x)+\boldsymbol{\Pi}_{\mathrm{e}}(x)+\boldsymbol{\Pi}_{\mathrm{B}}(x)\right]=0
$$

\footnotetext{
${ }^{3}$ Note that, when heat fluxes are neglected, the natural closure relations for the gyrotropic pressure components would be provided by the double-adiabatic law (Chew et al. 1956) (see, e.g. also Hau et al. 1993; Hau 2002, for convenient formulation and extensions). In the case considered here of incompressible flow, no heat fluxes and no gradients parallel to the magnetic field, the double-adiabatic relations and the dynamical pressure equations in the eTF model are equivalent to two different polytropic relations for $p_{\|}$and $p_{\perp}$, namely $\gamma_{\perp}=2$ and $\gamma_{\|}=1$ (see, e.g. Cerri 2012; Cerri et al. 2014; Del Sarto \& Pegoraro 2018).
} 
where $\boldsymbol{\Pi}_{\mathrm{B}} \equiv\left(B^{2} / 8 \pi\right) \boldsymbol{I}-\boldsymbol{B} \boldsymbol{B}$ is the magnetic pressure tensor ( $\boldsymbol{I}$ being the identity tensor). Within an (anisotropic) MHD model of plasma, the shear flow does not play a role in the equilibrium profile. In fact, when $\pi_{\mathrm{p}}^{(1)}$ is neglected, the equilibrium condition for the above configuration simply consists of a balance between the magnetic pressure, $P_{B}(x)=B^{2}(x) / 8 \pi$, and the perpendicular thermal pressures, $P_{\perp}(x)=p_{\perp \mathrm{p}}(x)+p_{\perp \mathrm{e}}(x)$. In particular, that includes the widely adopted uniform and homogeneous plasma configuration, namely $p_{\perp \alpha}=p_{\| \alpha, 0}, p_{\perp \alpha}=p_{\perp \alpha, 0}, B_{y}=B_{0 y}$ and $B_{z}=B_{0 z}$, that is not allowed anymore when FLR corrections (or the full pressure-tensor equations) are included in the fluid description (Cerri 2012; Cerri et al. 2013, 2014). In general, the solution of the MHD equilibrium condition is completely described by the chosen magnetic profile in (2.5), which determines all the profiles of the other relevant quantities. Let us now consider the changes of a given MHD equilibrium profile that are induced by a velocity shear of the type described above when first-order FLR corrections are taken into account. In this case, the only component of $\pi_{\mathrm{p}}^{(1)}$ that is relevant to the equilibrium condition is

$$
\pi_{\mathrm{p}, x x}^{(1)}=-\frac{1}{2} \frac{p_{\perp \mathrm{p}}}{\Omega_{c \mathrm{p}}}\left(b_{z} \frac{\mathrm{d} u_{y}}{\mathrm{~d} x}-b_{y} \frac{\mathrm{d} u_{z}}{\mathrm{~d} x}\right) .
$$

From (2.7), one directly identifies the connection between the fluid vorticity, $\omega \equiv \nabla \times$ $\boldsymbol{u}$, and the magnetic field direction $\boldsymbol{b}$, arising as a consequence of the FLR effects:

$$
\pi_{\mathrm{p}, x x}^{(1)}=-\frac{1}{2} \frac{p_{\perp \mathrm{p}}}{\Omega_{c \mathrm{p}}}(\boldsymbol{b} \cdot \boldsymbol{\omega}) \longrightarrow \frac{\mathrm{d}}{\mathrm{d} x}\left[\left(1-\frac{m_{\mathrm{p}} c}{e B} \frac{\boldsymbol{b} \cdot \boldsymbol{\omega}}{2}\right) p_{\perp \mathrm{p}}+p_{\perp \mathrm{e}}+\frac{B^{2}}{8 \pi}\right]=0,
$$

where $\omega_{y}=-u_{z}^{\prime}$ and $\omega_{z}=u_{v}^{\prime}$ are the components of the fluid vorticity in our configuration. Therefore, the FLR corrections give rise to an intrinsic asymmetry in the system's configurations, pressure anisotropy (and most likely also the subsequent dynamics), which depends on the degree of alignment (or anti-alignment) between the flow vorticity and the magnetic field, namely on the sign of $\boldsymbol{b} \cdot \boldsymbol{\omega}$. Such asymmetry has been highlighted in previous numerical simulations and analytical studies (see, e.g. Nagano 1978; Hazeltine, Hsu \& Morrison 1987; Cai et al. 1990; Huba 1996; Ramos 2005b; Nakamura et al. 2010; Cerri et al. 2013; Henri et al. 2013; Del Sarto et al. 2016, 2017; Franci et al. 2016; Parashar \& Matthaeus 2016; Yang et al. 2017; Del Sarto \& Pegoraro 2018). We stress, however, that the simple dependence on $\boldsymbol{\omega}$ and $\boldsymbol{b}$ in (2.7) is related to the simplified character of the configuration considered here.

Now assume that $\mathcal{F}_{\perp}(x), \mathcal{G}_{\perp}(x)$ and $\mathcal{H}(x)$ are the solutions for the anisotropic MHD equilibrium describing the profiles of the proton perpendicular pressure, $p_{\perp \mathrm{p}}=p_{\perp \mathrm{p}, 0} \mathcal{F}_{\perp}(x)$, of the electron perpendicular pressure, $p_{\perp \mathrm{e}}=p_{\perp \mathrm{e}, 0} \mathcal{G}_{\perp}(x)$, and of the magnetic pressure, $P_{B}(x)=\left(B_{0}^{2} / 8 \pi\right) \mathcal{H}(x)$ (here $p_{\perp \mathrm{p}, 0}, p_{\perp \mathrm{e}, 0}$ and $B_{0}$ are the asymptotic constant values of the pressures and of the magnetic field away from the shear layer, on one of the two sides - here we do not assume a symmetric shear layer; see appendix A for details). We now seek FLR-corrected equilibrium profiles in the form $\widetilde{\mathcal{F}}_{\perp}(x)=\mathcal{F}_{\perp}(x) f_{\perp}(x), \widetilde{\mathcal{G}}_{\perp}(x)=\mathcal{G}_{\perp}(x) g_{\perp}(x)$ and $\widetilde{\mathcal{H}}(x)=\mathcal{H}(x) h(x)$, where $f_{\perp}, g_{\perp}$ and $h$ are the 'correction functions'. By requiring quasi-neutrality and that the MHD profile $\beta_{\perp \mathrm{p}}(x)$ does not change when passing to the corresponding FLR-corrected profile, the solution can be given in term of one function only, i.e. $f_{\perp}(x)=g_{\perp}(x)=h(x)$ (see appendix A):

$$
f_{\perp}(x)=\left\{\frac{\widetilde{U}^{\prime}(x)}{2}+\sqrt{1+\left(\frac{\widetilde{U}^{\prime}(x)}{2}\right)^{2}}\right\}^{2},
$$


where we have defined

$$
\widetilde{U}^{\prime}(x) \equiv \frac{\widetilde{\beta}_{\perp \mathrm{p}, 0}}{2} \frac{m_{\mathrm{p}} c}{e B_{0}} \frac{\mathcal{F}_{\perp}(x)}{\mathcal{H}(x)}\left(\frac{B_{0 z}}{B_{0}} H_{z}(x) u_{y}^{\prime}(x)-\frac{B_{0 y}}{B_{0}} H_{y}(x) u_{z}^{\prime}(x)\right),
$$

with $\widetilde{\beta}_{\perp \mathrm{p}, 0} \equiv \beta_{\perp \mathrm{p}, 0} /\left(1+\beta_{\perp, 0}\right)$ for brevity. Note that the solution in (2.9) has been obtained taking into account the FLR corrections computed with the self-consistent (i.e. FLR-corrected) equilibrium magnetic field profile, $B(x)=B_{0} \sqrt{\mathcal{H}(x) f_{\perp}(x)}$. The equilibrium profiles resulting from (2.9) are then naturally asymmetric with respect to the sign of $\boldsymbol{\omega} \cdot \boldsymbol{b}$.

\subsection{FLR profiles and approximate kinetic equilibria}

The profiles derived above can be used to initialize the ion distribution function in order to set-up an approximate kinetic equilibrium (see Cerri et al. 2013). For instance, assuming the inhomogeneity direction to be along $x$, the magnetic field to be in the $z$-direction, $\boldsymbol{B}=B_{z}(x) \boldsymbol{e}_{z}$ and the flow to be along the $y$-axis, $\boldsymbol{u}=u_{y}(x) \boldsymbol{e}_{y}$, one obtains the following temperatures:

$$
\begin{gathered}
T_{x}(x)=\frac{p_{\perp \mathrm{p}, 0}}{n_{0}}(1-\chi(x))\left(\mathcal{F}_{\perp}(x) f_{\perp}(x)\right)^{\left(\gamma_{\perp}-1\right) / \gamma_{\perp}}, \\
T_{y}(x)=\frac{p_{\perp \mathrm{p}, 0}}{n_{0}}(1+\chi(x))\left(\mathcal{F}_{\perp}(x) f_{\perp}(x)\right)^{\left(\gamma_{\perp}-1\right) / \gamma_{\perp}}, \\
T_{z}=T_{\| \mathrm{p}}=\frac{p_{\| \mathrm{p}, 0}}{n_{0}}\left(\mathcal{F}_{\perp}(x) f_{\perp}(x)\right)^{\left(\gamma_{\|}-1\right) / \gamma_{\perp}},
\end{gathered}
$$

from which the three thermal velocities, $v_{\mathrm{th}, x}(x), v_{\mathrm{th}, y}(x)$ and $v_{\mathrm{th}, z}(x)$ can be defined. The parameter $\chi$ is defined by the first-order FLR correction to the pressure tensor in (2.8), and provides the agyrotropy of the distribution as a function of the alignment between the flow vorticity, $\boldsymbol{\omega}$, and the self-consistent FLR-corrected magnetic field. In our transverse case with $\boldsymbol{u}=u_{y}(x) \boldsymbol{e}_{z}$ and $\boldsymbol{B}=B_{z}(x) \boldsymbol{e}_{z}$, it reads

$$
\chi(x) \equiv \frac{1}{2} \frac{m_{\mathrm{p}} c}{e|\boldsymbol{B}|}(\boldsymbol{\omega} \cdot \boldsymbol{b})=\frac{1}{2} \frac{m_{\mathrm{p}} c}{e B_{0}} \frac{u_{y}^{\prime}(x)}{H_{z}(x) \sqrt{f_{\perp}(x)}},
$$

where $u_{y}^{\prime}(x)=\mathrm{d} u_{y} / \mathrm{d} x$. The 'Maxwellian-like' particle distribution function corresponding to the above profiles reads

$$
F_{\mathrm{M}}^{(\mathrm{FLR})}\left(x, v_{x}, v_{y}, v_{z}\right)=\frac{(2 \pi)^{-3 / 2} n(x)}{\sqrt{T_{x}(x) T_{y}(x) T_{z}(x)}} \exp \left\{-\frac{v_{x}^{2}}{2 T_{x}(x)}-\frac{\left(v_{y}-u_{y}(x)\right)^{2}}{2 T_{y}(x)}-\frac{v_{z}^{2}}{2 T_{z}(x)}\right\} .
$$

Note that, in the general case, a distribution function reproducing the FLR-corrected profiles would be more complicated, since it may have to give non-diagonal pressure terms. Nevertheless, the equilibrium profiles derived from the FLR correction function $f_{\perp}(x)$ in (2.9) still holds for a generic flow and magnetic-field profile (given that they lie in the plane perpendicular to the inhomogeneity direction; see $\S$ A.1) and can be used to set up such 'Maxwellian-like' distributions. We stress anyway that a distribution function built in this way is only an approximate kinetic equilibrium, which nevertheless can strongly reduce the spurious fluctuations arising from a readjustment induced by adopting MHD-like equilibrium profiles within a kinetic (or a hybrid kinetic) framework. Unfortunately, exact solutions of the kinetic (or of the hybrid kinetic) problem usually need to consider simplified configurations, e.g. of 
the magnetic field, and/or cannot exactly constrain the resulting velocity profiles beforehand (see, e.g. Cai et al. 1990; Attico \& Pegoraro 1999; Mahajan \& Hazeltine 2000; Bobrova et al. 2001; Malara et al. 2018). Which solution is better to use clearly depends on the problem under consideration. For instance, in the context of the Earth's flank magnetopause we are dealing with inhomogeneous magnetic field and density profiles (and directions), so the approach presented here is more appropriate for that case.

\subsection{Readjustment time scale of unbalanced equilibria}

As mentioned in the Introduction, taking into account the leading kinetic effects (such as the above first-order ion-FLR correction) may be necessary already at the level of the initial plasma configuration. In fact, adopting an ideal MHD initial equilibrium in a kinetic framework will result in a quick readjustment and in the development of spurious large-amplitude fluctuations (see Cerri et al. 2013; Henri et al. 2013).

When MHD equilibria are employed in kinetic simulations where a sheared flow is present, the unbalanced leading ion-FLR corrections will induce a readjustment on time scales $\tau_{\pi}$ of the order ${ }^{4}$

$$
\tau_{\pi}^{-1} \sim \beta_{i, \perp}^{-1 / 2} M_{A}\left(\frac{\rho_{i}}{L_{u}}\right)^{2} \Omega_{c, i} \sim \beta_{i, \perp}^{1 / 2} M_{A}\left(\frac{d_{i}}{L_{u}}\right)^{2} \Omega_{c, i},
$$

where $M_{A} \equiv u_{0} / v_{A}$ and $L_{u}$ are the Alfvénic Mach number and length scale of the background shear flow. It may be useful to compare this readjustment time scale with the growth rate of the fastest-growing mode (FGM) for the Kelvin-Helmholtz instability,

$$
\gamma_{\mathrm{FGM}}^{(\mathrm{KHI})} \sim \frac{1}{4} k_{\mathrm{FGM}} u_{0} \sim 0.1 \beta_{i, \perp}^{-1 / 2} M_{A}\left(\frac{\rho_{i}}{L_{u}}\right) \Omega_{c, i} \sim 0.1 M_{A}\left(\frac{d_{i}}{L_{u}}\right) \Omega_{c, i},
$$

where we have used the relation $k_{\mathrm{FGM}} L_{u} \sim 0.4$ derived in the compressible MHD limit (see Faganello \& Califano 2017, and references therein). Therefore, the effects of such readjustment on the KHI growth are of order

$$
\gamma_{\mathrm{FGM}}^{(\mathrm{KHI})} \tau_{\pi} \sim 0.1\left(\frac{L_{u}}{\rho_{i}}\right) \sim 0.1 \beta_{i, \perp}^{-1 / 2}\left(\frac{L_{u}}{d_{i}}\right),
$$

which is typically smaller than (or of the order of) unity for the magnetopause case, meaning that any readjustment happens faster than the instability itself and therefore will strongly change the equilibrium on top of which the KHI develops.

A sketch of the behaviour of time scales in (2.16) and (2.18) with respect to the relevant parameters is provided in figure 1. MHD-like behaviour is recovered in the parameter space denoted by yellow/white colours.

\subsection{Sustainability of pressure agyrotropy}

An interesting feature of the interaction between the pressure tensor and a sheared flow is the sustainability and/or the generation of pressure 'agyrotropy' (Cerri et al. 2013,

\footnotetext{
${ }^{4}$ Here we are assuming that the corresponding electron-FLR corrections are negligible compared to those of the ions. This assumption may break down for $\beta_{e, \perp} \sim\left(m_{i} / m_{e}\right) \beta_{i, \perp} \gg \beta_{i, \perp}$.
} 

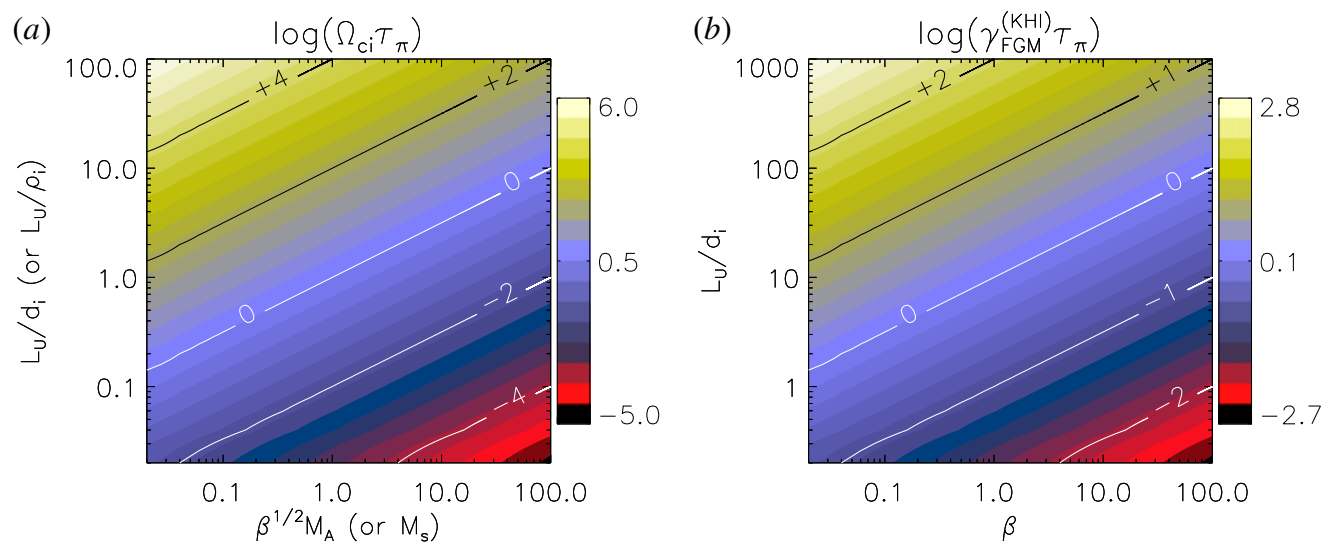

FIGURE 1. (a) Iso-surfaces of $\log \left(\Omega_{c \mathrm{i}} \tau_{\pi}\right)$ in the $L_{u} / d_{i}$ versus $\beta^{1 / 2} M_{A}$ plane. The same iso-surfaces apply to the $L_{u} / \rho_{i}$ versus $M_{s}$ plane $\left(M_{s} \equiv u_{0} / c_{s}\right.$ is the Mach number, $c_{s}$ being the sound speed). (b) Iso-surfaces of $\log \left(\gamma_{\mathrm{FGM}}^{(\mathrm{KHI})} \tau_{\pi}\right)$ in the $L_{u} / d_{i}$ versus $\beta$ plane.

2014; Del Sarto et al. 2016, 2017). This means that, in addition to the typical pressure anisotropy with respect to the magnetic-field direction that is typical of collisionless plasmas $\left(p_{\perp} \neq p_{\|}\right)$, now additional pressure anisotropy can be present in the plane perpendicular to $\boldsymbol{B}$, e.g. $p_{\perp, 1} \neq p_{\perp, 2} \neq p_{\|}$, where $\left(\boldsymbol{e}_{\perp, 1}, \boldsymbol{e}_{\perp, 2}, \boldsymbol{e}_{\|}\right)$is any orthogonal basis within which the pressure tensor is diagonal and where $\boldsymbol{e}_{\perp, 1}$ and $\boldsymbol{e}_{\perp, 2}$ define the plane perpendicular to the magnetic field. In this section we analyse this aspect in terms of equilibrium configurations and their corresponding agyrotropy. However, we stress that this feature has consequences for the dynamics of a collisionless plasma as well, e.g. modifying linear properties of perturbations (e.g. Del Sarto et al. 2016, 2017), enhancing the kinetic activity related to vorticity, current sheets, reconnection and energy transfer in turbulence (e.g. Greco et al. 2012; Servidio et al. 2012, 2014; Yang et al. 2017) and possibly affecting the regulation of anisotropies in accretion disks (e.g. Kunz, Stone \& Quataert 2016).

In our configuration it is easy to show that the FLR effects introduce an agyrotropy, $\Delta_{\perp}$, i.e. an anisotropy in the plane perpendicular to the magnetic field (see, e.g. Scudder \& Daughton 2008, for a general formulation), given by

$$
\Delta_{\perp}=\frac{|\boldsymbol{\omega} \cdot \boldsymbol{b}|}{\Omega_{c i}} \equiv|2 \chi| .
$$

Since only the first-order FLR corrections have been retained in the present description, only small deviations from gyrotropy are correctly described in this case, i.e. the condition $|\chi|=\left|\boldsymbol{\omega} \cdot \boldsymbol{b} / 2 \Omega_{c \mathrm{i}}\right| \ll 1$ should hold. Also, in this approximation the equilibrium exhibits an asymmetry with respect to the sign of $\boldsymbol{\omega} \cdot \boldsymbol{b}$, but $\Delta_{\perp}$ does not. In order to have such asymmetry in the agyrotropy, next-order corrections or the full pressure tensor must be retained. In the latter case, the agyrotropy in the plane perpendicular to $\boldsymbol{B}$ would be (Cerri et al. 2014)

$$
\Delta_{\perp}=\left|\frac{2 \chi}{1+\chi}\right|,
$$

where the condition $\chi \geqslant-1 / 2$ must hold because of the positivity constraint on pressure. 


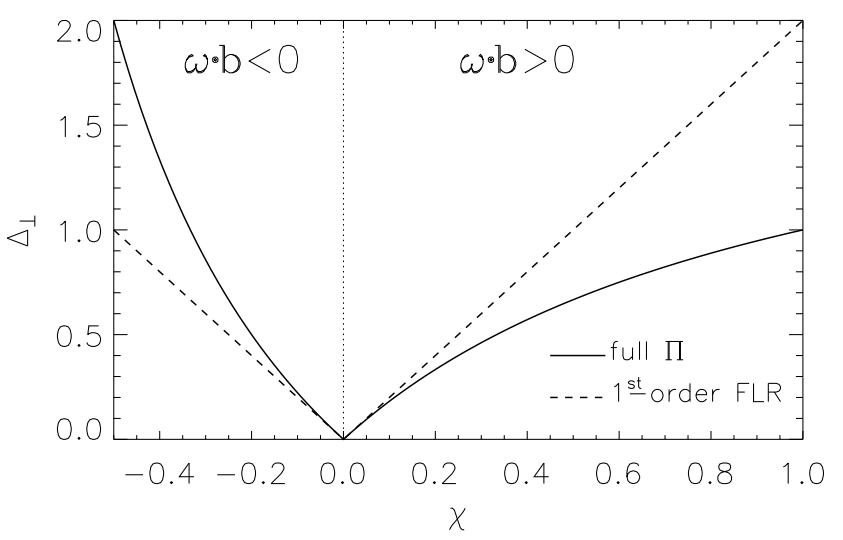

FIgURE 2. Pressure anisotropy in the plane perpendicular to the magnetic field direction, $\Delta_{\perp}$, versus $\chi \equiv \boldsymbol{\omega} \cdot \boldsymbol{b} / 2 \Omega_{c \text { i }}$ obtained from first-order FLR corrections (dashed line, equation (2.19)) and from the full pressure-tensor equation (continuous line, equation (2.20)). Positivity of pressure from the full- $\Pi$ treatment requires $\chi \geqslant-1 / 2$ (see Cerri et al. 2014), while the FLR treatment holds for $|\chi| \ll 1$.

In figure 2 we report a comparison between the pressure anisotropy in the plane perpendicular to the magnetic field, $\Delta_{\perp}$, as a function of the parameter $\chi$, obtained via the full pressure-tensor equation (Cerri et al. 2014) and via first-order FLR corrections.

\subsection{A broader view: relevance to other instabilities and turbulent environments}

As we will show in $\S 3$, the main consequences related to the ion-FLR effects reported in this paper have a direct effect in the current system of a planetary magnetopause. Moreover, these ion-kinetic effects can cause the asymmetric development of KHI at the dawn and the dusk sides of such magnetosphere, as well as other non-ideal features (see, e.g. Nagano 1978; Huba 1996; Terada et al. 2002; Nakamura et al. 2010; Henri et al. 2012; Masters et al. 2012; Sundberg et al. 2012; Taylor et al. 2012; Delamere et al. 2013; Paral \& Rankin 2013; Haaland et al. 2014; Johnson et al. 2014; Liljeblad et al. 2014; Walsh et al. 2014; Gershman et al. 2015; Gingell et al. 2015; De Camillis et al. 2016). However, ion-FLR effects and their relations with anisotropy, vorticity and current sheets can have implications on a wide variety of astrophysical and space scenarios.

In fact there are further shear-driven instabilities that may also get relevant feedback from anisotropy (and agyrotropy) developed (or sustained) by the underlying shear flow within a kinetic description such as, for instance, for the case of magnetorotational instability (MRI) in accretion disks (e.g. Ferraro 2007; Riquelme et al. 2012; Kunz et al. 2016; Squire, Quataert \& Kunz 2017b). Furthermore, ion-kinetic effects such as FLR and pressure-tensor dynamics can affect anisotropy-driven instabilities themselves (e.g. Schekochihin et al. 2010; Rosin et al. 2011; Sarrat, Del Sarto \& Ghizzo 2016; Squire et al. 2017a), which are relevant, e.g. in the evolution of the solar wind (e.g. Hellinger et al. 2006; Tenerani, Velli \& Hellinger 2017; Yoon 2017) and in magnetic reconnection (e.g. Schoeffler, Drake \& Swisdak 2011; Cassak et al. 2015).

Finally, current sheets and the associated reconnection processes are fundamental ingredients of turbulent plasmas (e.g. Matthaeus \& Lamkin 1986; Biskamp 2008; 
Servidio et al. 2010, 2011; Lazarian, Eyink \& Vishniac 2012; Karimabadi et al. 2013a; Servidio et al. 2015; Franci et al. 2016; Cerri et al. 2017). In this context, currents and coherent structures are typically related to simultaneous enhancement of vorticity, kinetic activity, turbulent transfer and dissipation (e.g. Servidio et al. 2012, 2014; Karimabadi et al. 2013b; Valentini et al. 2014, 2016; Wan et al. 2015; Franci et al. 2016; Parashar \& Matthaeus 2016; Yang et al. 2017; Grošelj et al. 2017; Camporeale et al. 2018; Sorriso-Valvo et al. 2018). Furthermore, reconnection/structures have been recently proved to enhance/trigger the kinetic turbulent cascades in real space (Cerri \& Califano 2017; Franci et al. 2017; Camporeale et al. 2018) and also to be related to simultaneous velocity-space cascades (Servidio et al. 2017; Cerri, Kunz \& Califano 2018; Pezzi et al. 2018). These reconnecting current sheets and the resulting magnetic structures are quasi-equilibrium pressure-balanced structures with embedded sheared flows even within a turbulent environment (see e.g. Cerri \& Califano 2017). Therefore ion-kinetic effects such as FLR contributions (or the full pressure tensor; see Cerri et al. (2014), Yang et al. (2017) and Del Sarto \& Pegoraro (2018)) may play a relevant role in the complex interplay between currents, vorticity, reconnection, non-Maxwellian features, velocity-space cascades and dissipation in turbulent plasmas.

\section{Application to the LLBL of the Earth's magnetopause}

Let us now consider an explicit application to the LLBL of the Earth's magnetopause, the goal being to show that the observed deviations from the ideal ChapmanFerraro current system highlighted in Haaland et al. (2014) can be qualitatively explained with the ion-FLR corrections. We want to stress that this is not meant to be a quantitative explanation of the observed profiles, since also the three-dimensional geometry and other effects may contribute to the actual profiles. In what follows, equations are normalized to the proton mass, inertial length and cyclotron frequency ( $m_{\mathrm{p}}, d_{\mathrm{p}}$ and $\Omega_{c \mathrm{p}}$, respectively), and the Alfvén speed $\left(v_{A}\right)$.

We consider a local one-dimensional model the LLBL region in which the inhomogeneity direction $(x)$ is perpendicular to the plane $(y z)$ where both the flow and the magnetic field lie. Typically, hyperbolic tangent give a reasonably realistic modelling of the flow,

$$
\begin{aligned}
& u_{y}(x)=u_{0} \sin \phi \tanh \left(\frac{x-x_{u, 0}}{L_{u}}\right), \\
& u_{z}(x)=u_{0} \cos \phi \tanh \left(\frac{x-x_{u, 0}}{L_{u}}\right),
\end{aligned}
$$

where $\phi$ is the angle between the $z$-axis and the plane where the sheared flow velocity lies, and of the magnetic field,

$$
\begin{aligned}
B_{y}(x)= & B_{0}\left\{\frac{B_{G}}{B_{0}} \sin \vartheta\left[1+\frac{\Delta B_{\|}}{2 B_{G}}\left(1-\tanh \left(\frac{x-x_{B, 0}}{L_{B}}\right)\right)\right]\right. \\
& \left.+\frac{\Delta B_{\perp}}{2 B_{0}} \cos \vartheta\left[1+\tanh \left(\frac{x-x_{B, 0}}{L_{B}}\right)\right]\right\}, \\
B_{z}(x)= & B_{0}\left\{\frac{B_{G}}{B_{0}} \cos \vartheta\left[1+\frac{\Delta B_{\|}}{2 B_{G}}\left(1-\tanh \left(\frac{x-x_{B, 0}}{L_{B}}\right)\right)\right]\right. \\
& \left.-\frac{\Delta B_{\perp}}{2 B_{0}} \sin \vartheta\left[1+\tanh \left(\frac{x-x_{B, 0}}{L_{B}}\right)\right]\right\},
\end{aligned}
$$


where $B_{0}=\sqrt{B_{G}^{2}+\Delta B_{\perp}^{2}}$ and $\vartheta$ is the angle between the $z$-axis and the magnetic field at $x \rightarrow-\infty^{5}$. The above magnetic profile accounts both for variations that are purely in magnitude, through $\Delta B_{\|}$, and for rotations (magnetic shear) of the magnetic-field direction, through $\Delta B_{\perp}$ (see, e.g. Fadanelli et al. 2018, for the effects of $\Delta B_{\perp}$ on KHI at the Earth's magnetospheric flanks). Note that usually $x_{u, 0}=x_{B, 0}$ and $L_{u}=L_{B}$ are assumed in numerical simulations (see, e.g. Miura 1987; Fujimoto \& Terasawa 1995; Otto \& Fairfield 2000; Nykyri \& Otto 2004; Nakamura \& Fujimoto 2005; Faganello, Califano \& Pegoraro 2008; Palermo et al. 2011; Tenerani et al. 2011; Faganello et al. 2012). However, recent satellite measurements have shown that the magnetic (and density) profiles can be slightly shifted with respect to the velocity shear and/or that the shear length scales of these quantities may differ, i.e. $x_{u, 0} \neq x_{n, 0}$ and/or $L_{u} \neq$ $L_{n}$ (Foullon et al. 2008; Haaland et al. 2014; Rossi 2015). This idea has been also recently implemented in numerical simulations in order to explain some observational features (Rossi 2015; Leroy \& Keppens 2017). Therefore, here we also take into account these features. For a magnetic profile as in (3.3)-(3.4) the MHD magnetic pressure function, $\mathcal{H}$, is given by

$$
\mathcal{H}(x)=\frac{B_{G}^{2}}{B_{0}^{2}}\left\{\left[1+\frac{\Delta B_{\|}}{2 B_{G}}\left(1-\tanh \left(\frac{x-x_{B, 0}}{L_{B}}\right)\right)\right]^{2}+\frac{\Delta B_{\perp}^{2}}{4 B_{G}^{2}}\left[1+\tanh \left(\frac{x-x_{B, 0}}{L_{B}}\right)\right]^{2}\right\},
$$

and the corresponding MHD thermal profiles are obtained in terms of

$$
\begin{aligned}
\mathcal{F}_{\perp}(x)=\mathcal{G}_{\perp}(x)= & 1+\frac{\Delta B_{\perp}^{2}}{\beta_{\perp, 0} B_{0}^{2}}-\frac{B_{G} \Delta B_{\|}}{\beta_{\perp, 0} B_{0}^{2}}\left[1-\tanh \left(\frac{x-x_{B, 0}}{L_{B}}\right)\right] \\
& -\frac{\Delta B_{\|}^{2}}{4 \beta_{\perp, 0} B_{0}^{2}}\left[1-\tanh \left(\frac{x-x_{B, 0}}{L_{B}}\right)\right]^{2} \\
& -\frac{\Delta B_{\perp}^{2}}{4 \beta_{\perp, 0} B_{0}^{2}}\left[1+\tanh \left(\frac{x-x_{B, 0}}{L_{B}}\right)\right]^{2},
\end{aligned}
$$

where $\beta_{\perp, 0} B_{0}^{2}=2 P_{\perp, 0} \equiv 2\left(p_{\perp \mathrm{p}, 0}+p_{\perp \mathrm{e}, 0}\right)$ and the positivity condition on pressure (see (A 10) in § A.3) here reads as

$$
B_{G} \Delta B_{\|}+\frac{\Delta B_{\|}^{2}}{2} \leqslant P_{\perp, 0}+\frac{\Delta B_{\perp}^{2}}{2} .
$$

The FLR corrections to the above MHD profiles are then given in terms of

$$
\begin{aligned}
\widetilde{U}^{\prime}(x)= & \frac{\widetilde{\beta}_{\perp \mathrm{p}, 0}}{2} \frac{u_{0}}{B_{0} L_{u}} \frac{\mathcal{F}_{\perp}(x)}{\mathcal{H}(x)} \cosh ^{-2}\left(\frac{x-x_{u, 0}}{L_{u}}\right) \\
& \times\left\{\frac{B_{G}}{B_{0}}\left[1+\frac{\Delta B_{\|}}{2 B_{G}}\left(1-\tanh \left(\frac{x-x_{B, 0}}{L_{B}}\right)\right)\right] \sin (\phi-\vartheta)\right. \\
& \left.-\frac{\Delta B_{\perp}}{2 B_{0}}\left[1+\tanh \left(\frac{x-x_{B, 0}}{L_{B}}\right)\right] \cos (\phi-\vartheta)\right\},
\end{aligned}
$$

which is again related to the sign of the scalar product between the fluid vorticity and the magnetic field through the $\sin (\phi-\vartheta)$ and $\cos (\phi-\vartheta)$ coefficients.

\footnotetext{
${ }^{5}$ The corresponding angle $\varphi$ between the $z$-axis and $\boldsymbol{B}$ at $x \rightarrow \infty$ is related to $\vartheta$ and $\Delta B_{\perp}$ by $\tan \varphi=$ $\left(\tan \vartheta+\Delta B_{\perp} / B_{G}\right) /\left(1-\tan \varphi \Delta B_{\perp} / B_{G}\right)$, and $\varphi=\vartheta$ when $\Delta B_{\perp}=0$.
} 


\begin{tabular}{|c|c|c|c|c|c|c|c|c|c|c|c|}
\hline \multirow[t]{2}{*}{ Case } & \multicolumn{3}{|c|}{ Flow parameters } & \multicolumn{4}{|c|}{ Magnetic-field parameters } & \multicolumn{4}{|c|}{ Plasma thermal parameters } \\
\hline & $u_{0}$ & $L_{u}$ & $x_{u, 0}$ & $\overline{\Delta B_{\|}}$ & $\Delta B_{\perp}$ & $L_{B}$ & $x_{B, 0}$ & $\beta_{\perp, 0}$ & $\beta_{\|, 0}$ & $\gamma_{\perp}$ & $\gamma_{\|}$ \\
\hline & & 2 & \pm 1 & 0.5 & 0.6 & 6 & 0 & 2 & 2 & 2 & 1 \\
\hline & \pm 2 & 2 & \pm 1 & 0.7 & 0.7 & 6 & 0 & 4 & 4 & 2 & 1 \\
\hline
\end{tabular}

TABLE 1. Summary of the parameters used for profiles in figure 3. All the parameters are normalized with respect to quantities characteristic of the SW region: flow speed is in $v_{\mathrm{A}}^{(\mathrm{SW})}$ units, lengths are in $d_{\mathrm{i}}^{(\mathrm{SW})}$ units and magnetic-field variations are in $B_{0}^{(\mathrm{SW})}=1$ units (from which $B_{G}=\sqrt{1-\Delta B_{\perp}^{2}}$ follows). The plus and minus sign in $u_{0}$ and in $x_{u, 0}$ are for the dusk and for the dawn side, respectively. We also remind the reader that $\vartheta=0$ and $\phi=\pi / 2$ in both cases.

\subsection{Current profiles at the Earth's flank magnetopause: an example}

Let us now consider a few explicit examples relevant for the magnetopause layer and see how the first-order FLR corrections qualitatively modify its current profile. For the sake of simplicity, we consider the case of $\vartheta=0$ and $\phi=\pi / 2$ and two slightly different regimes are taken into account. A summary of the parameters adopted for the example profiles is given in table 1. These parameters are chosen so that they are as realistic as possible for the low-latitude flanks of the magnetopause (Haaland et al. 2014), and they are also able to somewhat emphasize some of the resulting features ${ }^{6}$.

In figure 3 we report the current profile arising from a simple MHD configuration, $J_{y}^{(\mathrm{MHD})}$ and $J_{z}^{(\mathrm{MHD})}$ (light blue dashed line and orange dot-dashed line, respectively), as well as the profile accounting for the first-order FLR corrections in (3.8), $J_{y}^{(\text {FLR })}$ and $J_{z}^{(\mathrm{FLR})}$ (blue and red solid lines, respectively). The MHD profiles of the dusk and of the dawn sides, apart from the sign, have the same shape, i.e. it is the classic Chapman-Ferraro current layer (Chapman \& Ferraro 1930). On the other hand, the corresponding FLR-corrected profiles of the dawn and of the dusk sides are qualitatively different. This is the effect of the ' $\omega \boldsymbol{b}$ asymmetry' intrinsically encoded in the FLR contributions. Furthermore, the current structure of the shear layer in this latter case is much more complex than the Chapman-Ferraro MHD layer. In fact, a double-peak feature asymmetrically arises in $J^{(\text {FLR })}$ on the two sides of the flank magnetopause and the different modification of the two components of the current results in adjacent current sheets with different current directions (see figure 4, where we report the $x$-dependence of the angle between $\boldsymbol{J}$ and the $z$-axis, $\alpha=\arctan \left(J_{y} / J_{z}\right)$, for the cases shown in figure 3). These three peculiar features, namely (i) the dusk-dawn asymmetry of the current layer, (ii) the double-peak feature in the current profiles and (iii) two (or more) adjacent current sheets having thickness of several ion Larmor radii and with different current directions, are qualitatively consistent with the Cluster observations reported in Haaland et al. (2014). Taking into account these FLR effects can also be a relevant starting point for explaining certain anomalies occurring during magnetopause distortions related to large-scale magnetosheath plasma jets (see, e.g. Dmitriev \& Suvorova 2012).

\footnotetext{
${ }^{6}$ For instance, the choice of $L_{B}=6 d_{i}$ is consistent with the mean thickness reported by Haaland et al. (2014) of $\simeq 18 \rho_{i}$ of the dawn side, whereas there is no explicit indication for the thickness of the velocity shear. In the present work, we have considered a velocity-shear layer that is thinner that the magnetic shear layer and that are slightly shifted with respect to each other, in agreement with some other Cluster observations (e.g. Foullon et al. 2008; Rossi 2015).
} 
(a)

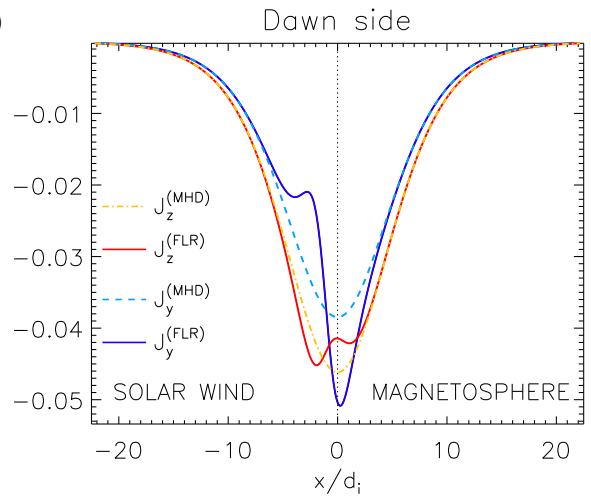

(c)

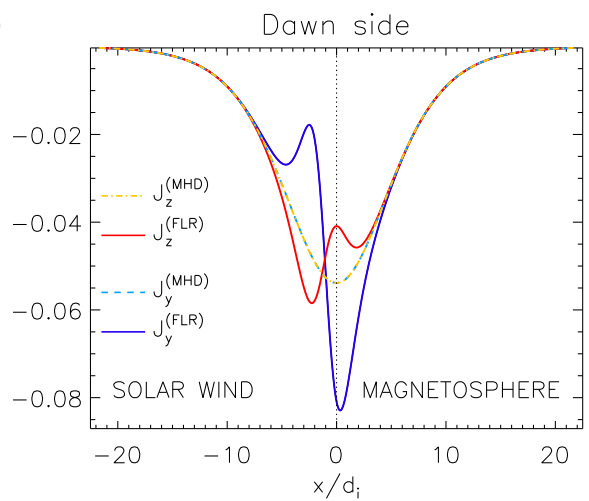

(b)

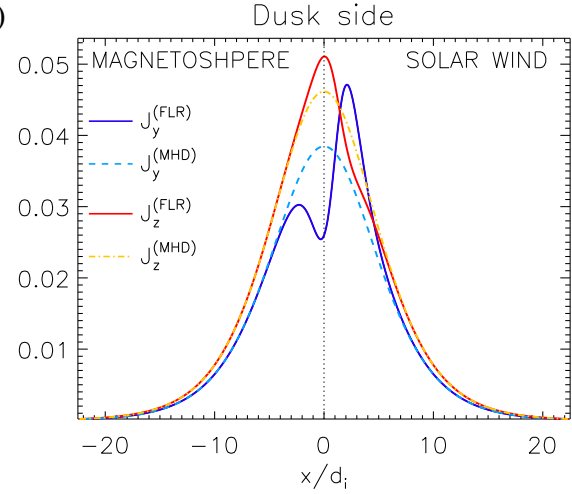

(d)

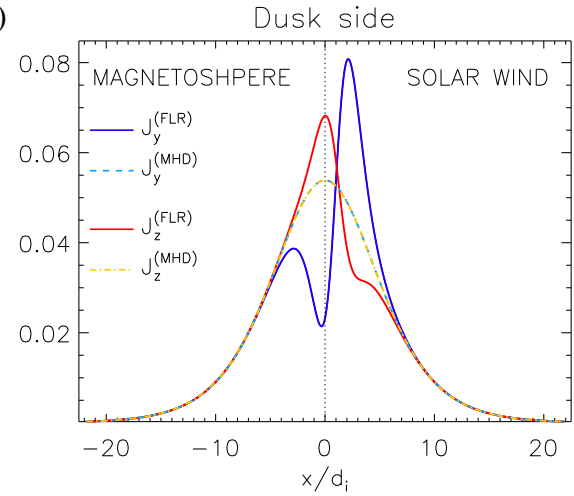

FIgURE 3. Current profiles for cases reported in table 1. (a,b) Case A, dawn $(a)$ and dusk $(b)$ sides. $(c, d)$ Case B, dawn $(c)$ and dusk $(d)$ sides. The MHD current profiles, $J_{y}^{(\mathrm{MHD})}$ and $J_{z}^{(\mathrm{MHD})}$, are reported with dashed light blue and dot-dashed orange lines, respectively, whereas the corresponding FLR-corrected profiles, $J_{y}^{(\mathrm{FLR})}$ and $J_{z}^{(\mathrm{FLR})}$, are drawn in blue and red solid lines, respectively.

Finally, we want to stress that here we focused on the FLR corrections to the magnetic and current structures, as most of the analysis done on satellite data for the purpose of reconstructing the characteristics of the Earth's flank magnetopause has been carried out in this direction. However, there are other relevant features and signatures of non-ideal effects that one could seek for in the available satellite data, as, for instance, the equilibrium profiles presented here would be supported by agyrotropic particle distribution functions localized in the large-scale shear-flow layer at the Earth's magnetopause ${ }^{7}$.

\section{Conclusions}

We have derived the one-dimensional equilibrium solutions for a shear-flow boundary layer within a so-called 'extended two-fluid' (eTF) model accounting for first-order ion finite-Larmor-radius (FLR) corrections in the double-adiabatic limit.

${ }^{7}$ Clearly, here we are not taking into account additional deviations from isotropy (and from pure gyrotropy) due to local current and vorticity sheets forming in a turbulent plasma (see, e.g. Servidio et al. 2012; Valentini et al. 2014, 2016; Franci et al. 2016; Cerri et al. 2018; Pezzi et al. 2018) and/or during reconnection events (see, e.g. Scudder \& Daughton 2008; Aunai, Hesse \& Kuznetsova 2013) 

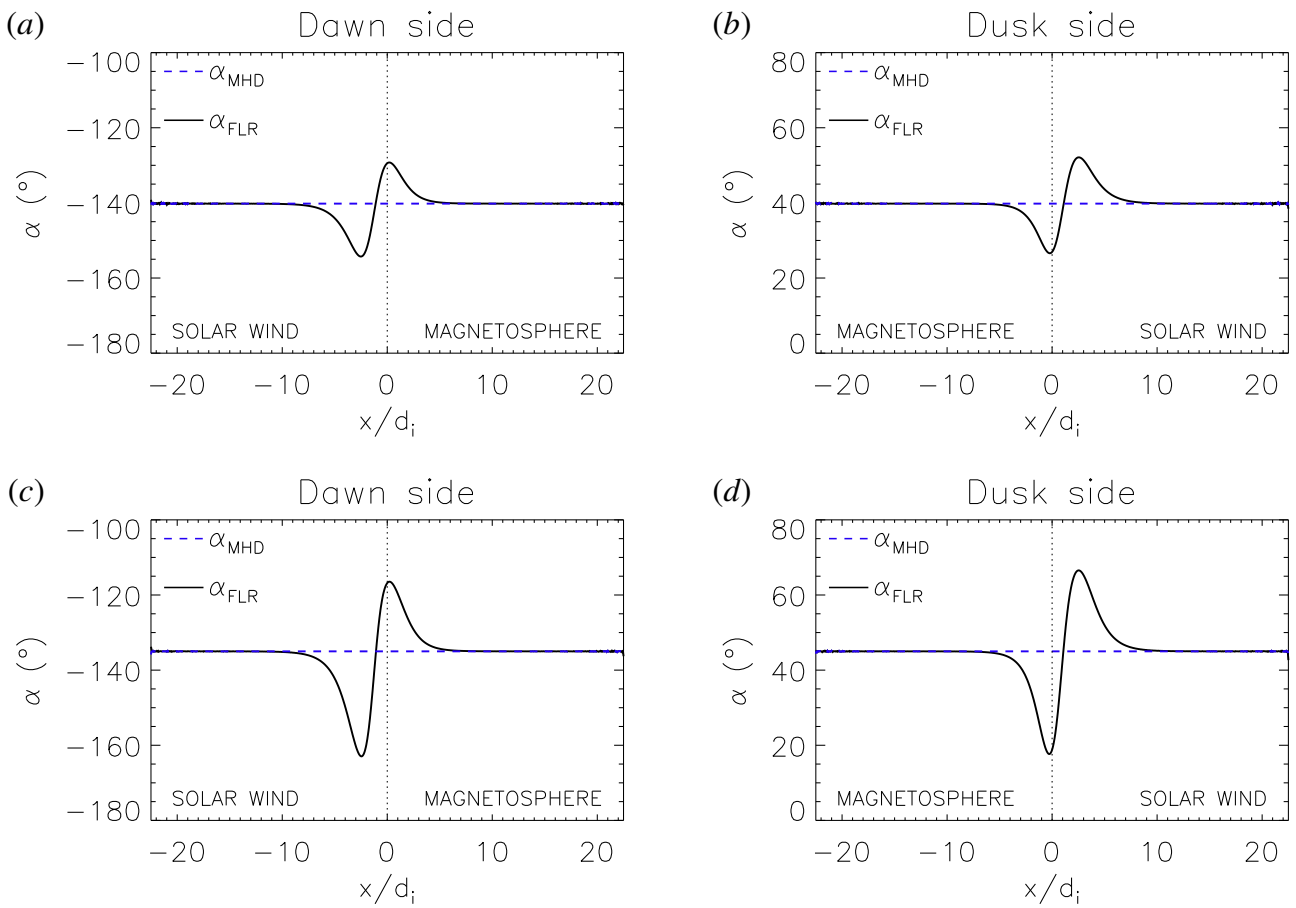

FIgURE 4. Profiles of the angle between the current $\boldsymbol{J}$ and the $z$-axis, $\alpha$, versus $x$ for cases reported in figure 3. (a) Case A, dawn $(a, b)$ and dusk $(b)$ sides. $(c, d)$ Case $\mathrm{B}$, dawn $(c)$ and dusk $(d)$ sides.

These analytical solutions represent a generalization of the solutions given in Cerri et al. 2013.

We have explicitly shown that first-order FLR corrections exhibit what we have called ' $\boldsymbol{\omega} \boldsymbol{b}$ asymmetry', i.e. an asymmetry that depends on the relative orientation of the fluid vorticity, $\boldsymbol{\omega}$, and of the magnetic-field direction, $\boldsymbol{b}$, through the scalar product $\boldsymbol{\omega} \cdot \boldsymbol{b}$. Moreover, depending again on the parameter $\boldsymbol{\omega} \cdot \boldsymbol{b}$, it has been demonstrated that the free energy available in the shear flow is able to develop and sustain a nonnegligible level of agyrotropy, i.e. a pressure (and temperature) anisotropy that is not limited to the directions parallel and perpendicular to the magnetic field (the so-called gyrotropy), but that manifests also within the plane perpendicular to $\boldsymbol{b}$ as $p_{\|} \neq p_{\perp, 1} \neq$ $p_{\perp, 2}$.

Finally, we have applied these FLR-corrected equilibrium profiles to few cases with parameters typical of the low-latitude flanks of the Earth's magnetopause. The resulting current structure has been shown to be more complex than the MHD layer by Chapman \& Ferraro (1930), in qualitative agreement with the Cluster observations recently reported in Haaland et al. (2014). In particular, by accounting for ion-FLR effects, we have been able to qualitatively reproduce the following key observational features: (i) an asymmetry of the current layer with respect to the dusk and the dawn sides of the magnetopause, (ii) a double-peak feature arising in the current profiles and (iii) the presence of adjacent current sheets having thickness of several ion Larmor radii and with different current directions. We want to stress that other effects that may contribute to further corrections have been neglected, e.g. the full ion pressure-tensor dynamics and the electron kinetic effects, so a quantitative comparison 
between the Cluster data and our profiles would be beyond the scope of the present work. Nevertheless, the good qualitative agreement between our one-dimensional analytical profiles and the Cluster observations reported in Haaland et al. (2014) shows that ion-FLR corrections are a relevant ingredient to correctly describe the Earth's flank magnetopause layer. Further effects, including a three-dimensional treatment of the magnetosphere-wind interface, as well as the full ion pressure tensor and self-consistent electron kinetic effects, will clearly have to be considered for a more quantitative comparison. In this regard, new and future space missions will also provide better measurements of the Earth's magnetopause structure and allow for a deeper understanding of the relevant plasma physics at play.

Finally, we underline that the main consequences of the ion-FLR effects reported in this work, and their relation to anisotropy, agyrotropy, vorticity and current sheets, may have implications for a wide variety of astrophysical and space collisionless plasmas, from the turbulent solar wind to low-luminosity accretion flows around compact objects.

\section{Acknowledgements}

The author acknowledges F. Pegoraro, F. Califano, D. Del Sarto and A. Tenerani for many valuable discussions on the subject in the past years, as well as M. W. Kunz for providing comments on the manuscript and the anonymous referees for comments that improved the clarity of the manuscript. This work was completed while S.S.C. was supported by the National Aeronautics and Space Administration under grant no. NNX16AK09G issued through the Heliophysics Supporting Research Program.

\section{Appendix A. Derivation of the equilibrium configurations of a shear-flow layer with FLR effects}

We now consider the case of a velocity-shear layer separating, for instance, two different plasmas. For the sake of simplicity, here we consider the one-dimensional equilibrium problem, which can be seen as a local approximation of the LLBL. A class of analytical solutions to the one-dimensional case that generalize the results provided in Cerri et al. (2013) and that include a much wider range of configurations of interest for what concerns magnetospheric observations will be provided.

\section{A.1. Preliminaries and assumptions}

In the following, we consider a given $x$-dependent incompressible MHD flow in the $y-z$ plane,

$$
\boldsymbol{u}=u_{y}(x) \boldsymbol{e}_{y}+u_{z}(x) \boldsymbol{e}_{z}, \quad \nabla \cdot \boldsymbol{u}=0,
$$

such that it becomes constant at the boundaries,

$$
\lim _{x \rightarrow \pm \infty} u_{y}(x)=u_{0 y}^{( \pm)}, \quad \lim _{x \rightarrow \pm \infty} u_{z}(x)=u_{0 z}^{( \pm)},
$$

i.e. we consider a localized velocity shear (the vorticity is vanishing at the boundaries, $\lim _{x \rightarrow \pm \infty} \nabla \times \boldsymbol{u}=0$ ). The magnetic field also lies on the $y-z$ plane,

$$
\boldsymbol{B}(x)=B_{0 y} H_{y}(x) \boldsymbol{e}_{y}+B_{0 z} H_{z}(x) \boldsymbol{e}_{z} .
$$

The associated magnetic pressure is

$$
P_{B}(x)=\frac{B_{0}^{2}}{8 \pi} \mathcal{H}(x), \quad \mathcal{H}(x) \equiv \frac{B_{0 y}^{2}}{B_{0}^{2}} H_{y}^{2}(x)+\frac{B_{0 z}^{2}}{B_{0}^{2}} H_{z}^{2}(x) \geqslant 0 \quad \forall x,
$$


where we have defined $B_{0}$ as the (constant) value of $|\boldsymbol{B}|$ at the right boundary ( $x \rightarrow$ $+\infty)$ :

$$
B_{0} \equiv \lim _{x \rightarrow+\infty} \sqrt{B_{0 y}^{2} H_{y}^{2}(x)+B_{0 z}^{2} H_{z}^{2}(x)}, \quad \lim _{x \rightarrow+\infty} \mathcal{H}(x)=1 .
$$

We further assume a polytropic relation for the thermal pressures ${ }^{8}$ :

$$
p_{\perp \mathrm{p}}=p_{\perp \mathrm{p}, 0} \mathcal{F}_{\perp}(x)=p_{\perp \mathrm{p}, 0}\left(\frac{n(x)}{n_{0}}\right)^{\gamma_{\perp \mathrm{p}}}, \quad p_{\perp \mathrm{e}}=p_{\perp \mathrm{e}, 0} \mathcal{G}_{\perp}(x)=p_{\perp \mathrm{e}, 0}\left(\frac{n(x)}{n_{0}}\right)^{\gamma_{\perp \mathrm{e}}}, \quad(\mathrm{A} 6 a, b)
$$

and

$$
p_{\| \mathrm{p}}=p_{\| \mathrm{p}, 0} \mathcal{F}_{\|}(x)=p_{\| \mathrm{p}, 0}\left(\frac{n(x)}{n_{0}}\right)^{\gamma_{\| \mathrm{p}}}, \quad p_{\| \mathrm{e}}=p_{\| \mathrm{e}, 0} \mathcal{G}_{\|}(x)=p_{\| \mathrm{e}, 0}\left(\frac{n(x)}{n_{0}}\right)^{\gamma_{\| \mathrm{e}}},
$$

where $\mathcal{F}_{\perp}, \mathcal{F}_{\|}, \mathcal{G}_{\perp}$ and $\mathcal{G}_{\|}$are functions that reduce to unity for $x \rightarrow+\infty$, as it is for $\mathcal{H}$.

\section{A.2. General one-dimensional MHD solutions for incompressible flows}

Within an (anisotropic) MHD model of plasma, the shear flow does not play a role in the equilibrium profile. In fact, when $\pi_{\mathrm{p}}^{(1)}$ is neglected, the equilibrium condition for the above configuration simply consists of a balance between the magnetic pressure, $B^{2}(x) / 8 \pi$, and total perpendicular thermal pressures, $P_{\perp}(x)$ :

$$
\frac{\mathrm{d}}{\mathrm{d} x}\left[p_{\perp \mathrm{p}}(x)+p_{\perp \mathrm{e}}(x)+\frac{B^{2}(x)}{2}\right]=0 .
$$

In particular, the above condition allows also the widely adopted uniform and homogeneous plasma configuration: $p_{\perp \alpha}=p_{\| \alpha, 0}, p_{\perp \alpha}=p_{\perp \alpha, 0}, B_{y}=B_{0 y}$ and $B_{z}=B_{0 z}$. Such homogeneous profiles are not an equilibrium solution when FLR corrections (or the full pressure-tensor equations) are included in the fluid description (Cerri 2012; Cerri et al. 2013, 2014), unless the velocity profile is a linear function of $x$ (see $\S$ A.3). In general, the solution of the MHD equilibrium condition in (A 8) is completely described by the magnetic pressure profile in (A 4), which determines all the other relevant functions, $\mathcal{F}_{\perp}(x)$ and $\mathcal{G}_{\perp}(x)$. In fact, assuming $\gamma_{\perp \mathrm{e}}=\gamma_{\perp \mathrm{p}}$ for simplicity, quasi-neutrality reads as

$$
\mathcal{G}_{\perp}(x)=\mathcal{F}_{\perp}(x)
$$

and the equilibrium condition finally gives $\mathcal{F}_{\perp}$ as function of $\mathcal{H}$,

$$
\mathcal{F}_{\perp}(x)=1+\frac{1}{\beta_{\perp, 0}}[1-\mathcal{H}(x)]
$$

where $\beta_{\perp, 0}=\beta_{\perp \mathrm{p}, 0}+\beta_{\perp \mathrm{e}, 0}$ (with $\beta_{\perp \alpha, 0} \equiv 8 \pi p_{\perp \alpha, 0} / B_{0}^{2}$ ), and the constant is set to $1+\beta_{\perp, 0}$ by the boundary conditions at $x \rightarrow+\infty$ (the requirement $\mathcal{F}_{\perp}(x) \rightarrow 1$ for $x \rightarrow+\infty$ is then automatically satisfied due to (A 5)). Furthermore, since the function $\mathcal{F}_{\perp}(x)$ is

\footnotetext{
${ }^{8}$ Note that in the case considered here of incompressible flow, no heat fluxes and no gradients parallel to the magnetic field, the double-adiabatic relations and the dynamical pressure equations in the eTF model are equivalent to two different polytropic relations for $p_{\|}$and $p_{\perp}$ (see, e.g. Cerri 2012; Cerri et al. 2014; Del Sarto \& Pegoraro 2018)
} 
related to the thermal pressure, it cannot assume negative values, which provides the additional condition

$$
\mathcal{F}_{\perp}(x) \geqslant 0 \quad \forall x \quad \Longleftrightarrow \mathcal{H}(x) \leqslant 1+\beta_{\perp, 0} .
$$

This states physically that any variation of the magnetic pressure, $\Delta B^{2} / 8 \pi=\left(B^{2}(x)-\right.$ $\left.B_{0}^{2}\right) / 8 \pi$, cannot exceed the total thermal pressure, $P_{\perp, 0}=p_{\perp \mathrm{p}, 0}+p_{\perp \mathrm{e}, 0}$, where $B_{0}, p_{\perp \mathrm{p}, 0}$ and $p_{\perp \mathrm{e}, 0}$ are the values at $x \rightarrow+\infty$. The parallel thermal pressures follow from the polytropic assumption, e.g. $\mathcal{F}_{\|}(x)=\left[\mathcal{F}_{\perp}(x)\right]^{\gamma_{\| p} / \gamma_{\perp \mathrm{p}}}$. Analogously, the temperature profiles follow from $T_{\perp \alpha}=p_{\perp \alpha} / n$ and $T_{\| \alpha}=p_{\| \alpha} / n$.

Starting from this MHD class of solutions, we self-consistently derive the corresponding equilibrium profiles with first-order FLR corrections.

\section{A.3. General first-order FLR corrections to the one-dimensional MHD solutions}

Let us now consider the changes to the MHD equilibrium profiles derived above that are induced by the velocity shear in (A 1) when first-order FLR corrections are taken into account. In this case, the only component of $\pi_{\mathrm{p}}^{(1)}$ that is relevant to the equilibrium condition is

$$
\pi_{\mathrm{p}, x x}^{(1)}=-\frac{1}{2} \frac{m_{\mathrm{p}} c}{e|\boldsymbol{B}|}\left(b_{z} \frac{\mathrm{d} u_{y}}{\mathrm{~d} x}-b_{y} \frac{\mathrm{d} u_{z}}{\mathrm{~d} x}\right) p_{\perp \mathrm{p}} .
$$

The equilibrium condition in (A 8) now reads

$$
\frac{\mathrm{d}}{\mathrm{d} x}\left\{\left[1-\frac{1}{2} \frac{B_{z}(x) u_{y}^{\prime}(x)-B_{y}(x) u_{z}^{\prime}(x)}{e B^{2}(x) / m_{\mathrm{p}} c}\right] p_{\perp \mathrm{p}}(x)+p_{\perp \mathrm{e}}(x)+\frac{B^{2}(x)}{8 \pi}\right\}=0,
$$

where the prime denotes the $x$-derivative. The above expressions can be explicitly written in terms of the fluid vorticity, $\boldsymbol{\omega} \equiv \nabla \times \boldsymbol{u}$, and of the magnetic field direction, $\boldsymbol{b}$ :

$$
\pi_{\mathrm{p}, x x}^{(1)}=-\frac{1}{2} \frac{m_{\mathrm{p}} c}{e B}(\boldsymbol{b} \cdot \boldsymbol{\omega}) p_{\perp \mathrm{p}} \quad \longrightarrow \quad \frac{\mathrm{d}}{\mathrm{d} x}\left[\left(1-\frac{m_{\mathrm{p}} c}{e B} \frac{\boldsymbol{b} \cdot \boldsymbol{\omega}}{2}\right) p_{\perp \mathrm{p}}+p_{\perp \mathrm{e}}+\frac{B^{2}}{8 \pi}\right]=0,
$$

where $\omega_{y}=-u_{z}^{\prime}$ and $\omega_{z}=u_{y}^{\prime}$ are the components of the fluid vorticity in our configuration. The dependence on $\boldsymbol{b} \cdot \boldsymbol{\omega}$ highlights the intrinsic asymmetry in the system due to FLR corrections and related to the degree of alignment (or anti-alignment) between the flow vorticity and the magnetic field. We stress, however, that the simple dependence on the vorticity and magnetic-field direction in (A 12) is due to the one-dimensional character of the problem considered here.

We now seek FLR-corrected equilibrium profiles in the form $\widetilde{\mathcal{F}}_{\perp}(x)=\mathcal{F}_{\perp}(x) f_{\perp}(x)$, $\widetilde{\mathcal{G}}_{\perp}(x)=\mathcal{G}_{\perp}(x) g_{\perp}(x)$ and $\widetilde{\mathcal{H}}(x)=\mathcal{H}(x) h(x)$, where $f_{\perp}, g_{\perp}$ and $h$ are the 'correction functions'. Due to the boundary conditions on the MHD flow, (A 2), the gyroviscous tensor vanishes at the boundaries, $\lim _{x \rightarrow \pm \infty} \pi_{\mathrm{p}}^{(1)}=0$, and thus the correction functions must reduce to unity accordingly, $\lim _{x \rightarrow \pm \infty}\left\{f_{\perp}(x), g_{\perp}(x), h(x)\right\}=1$. Therefore, $\widetilde{\mathcal{F}}_{\perp}$, $\widetilde{\mathcal{G}}_{\perp}$ and $\widetilde{\mathcal{H}}$ reduce to the corresponding MHD profiles away from the shear layer, where the vorticity vanishes (or, in general, where the vorticity becomes uniform and homogeneous). Moreover, since we want to preserve quasi-neutrality, $\widetilde{\mathcal{F}}(x)=\widetilde{\mathcal{G}}(x)$ must hold and therefore, using (A 9), we obtain the condition

$$
g_{\perp}(x)=f_{\perp}(x) .
$$


In order to relate $h(x)$ and $f_{\perp}(x)$, we actually need to impose a further constraint on the equilibrium. Such a condition cannot be derived from first principles and would rather be driven by a physical interpretation of the problem under study. Here we provide a viable option based on the plasma beta parameter (see, e.g. Cerri et al. 2013, 2014, for examples about different constraints). Since the (thermal) Larmor radius is sensitive to the (perpendicular) plasma beta, a very reasonable constraint is to require that the MHD profile $\beta_{\perp \mathrm{p}}(x)$ does not change when passing to the corresponding FLR-corrected profile, i.e.

$$
\left.\beta_{\perp \mathrm{p}}(x)\right|_{\mathrm{MHD}}=\left.\beta_{\perp \mathrm{p}}(x)\right|_{\mathrm{MHD}+\mathrm{FLR}} \quad \Longrightarrow \quad h(x)=f_{\perp}(x) .
$$

Then, using the above relations and the boundary conditions at $x \rightarrow+\infty$ to set the integration constant to $1+\beta_{\perp, 0}$, from (A 13) we obtain the following equation for $f_{\perp}(x)$ :

$$
f_{\perp}(x)-\widetilde{U}^{\prime}(x) \sqrt{f_{\perp}(x)}-1=0,
$$

where we have defined

$$
\widetilde{U}^{\prime}(x) \equiv \frac{\widetilde{\beta}_{\perp \mathrm{p}, 0}}{2} \frac{m_{\mathrm{p}} c}{e B_{0}} \frac{\mathcal{F}_{\perp}(x)}{\mathcal{H}(x)}\left(\frac{B_{0 z}}{B_{0}} H_{z}(x) u_{y}^{\prime}(x)-\frac{B_{0 y}}{B_{0}} H_{y}(x) u_{z}^{\prime}(x)\right),
$$

with $\widetilde{\beta}_{\perp \mathrm{p}, 0} \equiv \beta_{\perp \mathrm{p}, 0} /\left(1+\beta_{\perp, 0}\right)$ for brevity. Note that the above equation for $f_{\perp}(x)$ has been obtained taking into account the FLR corrections computed with the self-consistent equilibrium magnetic-field profile, $B(x)=B_{0} \sqrt{\mathcal{H}(x) f_{\perp}(x)}$ (we remind that $h(x)=f_{\perp}(x)$ holds). Finally, since $p_{\perp \mathrm{p}}(x)$ must be a positive quantity, we require $f_{\perp}(x) \geqslant 0 \forall x$, so that the only physical solution of (A 17) is

$$
f_{\perp}(x)=\left\{\frac{\widetilde{U}^{\prime}(x)}{2}+\sqrt{1+\left(\frac{\widetilde{U}^{\prime}(x)}{2}\right)^{2}}\right\}^{2} .
$$

This correctly reduces to unity for vanishing FLR terms, $\widetilde{U}^{\prime} \rightarrow 0$, recovering the MHD profiles. The resulting FLR-corrected profiles are therefore given by

$$
\begin{gathered}
p_{\perp \mathrm{p}}(x)=p_{\perp \mathrm{p}, 0} \mathcal{F}_{\perp}(x) f_{\perp}(x), \quad p_{\| \mathrm{p}}(x)=p_{\| \mathrm{p}, 0}\left(\mathcal{F}_{\perp}(x) f_{\perp}(x)\right)^{\gamma_{\|} / \gamma_{\perp}}, \\
n(x)=n_{0}\left(\mathcal{F}_{\perp}(x) f_{\perp}(x)\right)^{1 / \gamma_{\perp}}, \\
B_{y}(x)=B_{0 y} H_{y}(x) \sqrt{f_{\perp}(x)}, \quad B_{z}(x)=B_{0 z} H_{z}(x) \sqrt{f_{\perp}(x)},
\end{gathered}
$$

from which the current density, $\boldsymbol{J}=\boldsymbol{\nabla} \times \boldsymbol{B}$, follows.

\section{Appendix B. Derivation of the first-order FLR contributions: a perturbative approach}

In this appendix, we provide a derivation of the finite-Larmor-radius corrections to the gyrotropic pressure tensor based on a perturbative expansion of the full pressure-tensor dynamic equation ${ }^{9}$. Further, we explicitly comment on the symmetry properties of the perturbed equations and the corresponding solutions, which has a direct relevance for many configurations with a velocity shear.

Note that in the remainder of this appendix we are going to drop the species index everywhere, except when it is needed (e.g. when the sign of the charge matters).

\footnotetext{
${ }^{9}$ For a derivation based on a perturbative expansion of the distribution function, see Macmahon (1965) or Schekochihin et al. (2010). Other classical derivations can be found in Yajima (1966), Ramos (2005b) or in Mjølhus (2009).
} 


\section{B.1. Perturbative expansion of the pressure-tensor equation}

Let us consider the dynamic equation for the full pressure tensor,

$$
\frac{\partial \Pi_{i j}}{\partial t}+\frac{\partial}{\partial x_{k}}\left(\Pi_{i j} u_{k}+Q_{i j k}\right)+\Pi_{i k} \frac{\partial u_{j}}{\partial x_{k}}+\Pi_{j k} \frac{\partial u_{i}}{\partial x_{k}}=\Omega_{c \alpha}\left(\epsilon_{i k l} \Pi_{j k}+\epsilon_{j k l} \Pi_{i k}\right) b_{l},
$$

where $\epsilon_{i j k}$ is the Levi-Civita symbol, and perturbatively expand it with respect to the small parameter

$$
\varepsilon \equiv \frac{\rho}{L} \sim \frac{\omega}{\Omega} \ll 1,
$$

where $\rho$ is the Larmor radius, $L$ is the typical length scale of variation of fluid quantities and $\omega \sim u / L$ is the characteristic frequency of the fluid dynamics. Here we adopt the so-called 'fast-dynamics ordering', $u \sim v_{\text {th }}$ (Macmahon 1965; Ramos 2005a; Cerri et al. 2013). Using dimensionless quantities denoted by a tilde ${ }^{10}$, equation (B 1) rewrites as

$$
\begin{aligned}
& \left(\epsilon_{i k l} \widetilde{\Pi}_{j k}+\epsilon_{j k l} \widetilde{\Pi}_{i k}\right) \widetilde{b}_{l} \\
& \quad=\varepsilon \frac{\sigma_{\alpha}}{|\widetilde{\boldsymbol{B}}|}\left[\frac{\partial \widetilde{\Pi}_{i j}}{\partial \widetilde{t}}+\frac{\partial}{\partial \widetilde{x}_{k}}\left(\widetilde{\Pi}_{i j} \widetilde{u}_{k}\right)+\widetilde{\Pi}_{i k} \frac{\partial \widetilde{u}_{j}}{\partial \widetilde{x}_{k}}+\widetilde{\Pi}_{j k} \frac{\partial \widetilde{u}_{i}}{\partial \widetilde{x}_{k}}+\frac{\partial \widetilde{Q}_{i j k}}{\partial \widetilde{x}_{k}}\right],
\end{aligned}
$$

where we have defined $\sigma_{\alpha} \equiv \operatorname{sign}\left(e_{\alpha}\right)$, i.e. the sign embedded in the cyclotron frequency, $\Omega_{c \alpha}=e_{\alpha} B_{0} / m_{\alpha} c=\sigma_{\alpha}\left|e_{\alpha}\right| B_{0} / m_{\alpha} c \equiv \sigma_{\alpha}\left|\Omega_{c \alpha}\right|$. We then expand the pressure tensor and heat-flux tensor in powers of $\varepsilon$, i.e.

$$
\widetilde{\Pi}_{i j}=\sum_{n=0}^{\infty} \varepsilon^{n} \widetilde{\Pi}_{i j}^{(n)} \quad \text { and } \quad \widetilde{Q}_{i j k}=\sum_{n=0}^{\infty} \varepsilon^{n} \widetilde{Q}_{i j k}^{(n)} .
$$

Hereafter, the tilde will be omitted for the sake of simplicity and all the quantities have to be understood as dimensionless. The $n$ th-order pressure-tensor equation then reads

$$
\mathcal{L}_{B}\left[\Pi_{i j}^{(n)}\right]=\mathcal{R}_{u}\left[\Pi_{i j}^{(n-1)}\right]+\mathcal{D}\left[Q_{i j(k)}^{(n-1)}\right],
$$

where we have introduced the following linear operators:

$$
\begin{gathered}
\mathcal{L}_{\boldsymbol{B}}[\boldsymbol{\Pi}] \equiv\{\boldsymbol{\Pi} \times \boldsymbol{b}\}^{(\mathrm{sym})} \\
\mathcal{R}_{u}[\boldsymbol{\Pi}] \equiv \frac{\mathrm{d} \boldsymbol{\Pi}}{\mathrm{d} t}+\boldsymbol{\Pi}(\boldsymbol{\nabla} \cdot \boldsymbol{u})+\{\boldsymbol{\Pi}: \nabla \boldsymbol{u}\}^{(\mathrm{sym})} \\
\mathcal{D}[\boldsymbol{Q}] \equiv \nabla \cdot \boldsymbol{Q},
\end{gathered}
$$

which contribute to the evolution of the pressure tensor by involving only $\boldsymbol{B}, \boldsymbol{u}$ and $\boldsymbol{Q}$, respectively $(\partial / \partial t+\boldsymbol{u} \cdot \nabla$ has been replaced by the Lagrangian time derivative $\mathrm{d} / \mathrm{d} t$ for shortness). The zero order, $n=0$, gives

$$
\left(\epsilon_{i l m} \Pi_{l j}^{(0)}+\epsilon_{j l m} \Pi_{l i}^{(0)}\right) b_{m}=0,
$$

\footnotetext{
${ }^{10} \mathrm{We}$ normalize all the quantities with respect to the mass, $m$, the thermal speed, $v_{\text {th }}$, and a reference density and magnetic field, $n_{0}$ and $B_{0}$, respectively: $n=n_{0} \widetilde{n}, B=B_{0} \widetilde{B}, u=v_{\mathrm{th}} \widetilde{u}, \Pi=m n_{0} v_{\mathrm{th}}^{2} \widetilde{\Pi}$ and $Q=m n_{0} v_{\mathrm{th}}^{3} \widetilde{Q}$. The derivatives, are normalized as $\partial / \partial x=L^{-1} \partial / \partial \widetilde{x}$ and $\partial / \partial t=\tau^{-1} \partial / \partial \widetilde{t}$, with the ordering $L / \tau \sim u \sim v_{\text {th }}$.
} 
that means that $\Pi_{i j}^{(0)}$ belongs to the kernel of the $\mathcal{L}_{\boldsymbol{B}}$ operator, whereas the first-order equation, $n=1$, is

$$
\left(\epsilon_{i l m} \Pi_{l j}^{(1)}+\epsilon_{j l m} \Pi_{l i}^{(1)}\right) b_{m}=\frac{\sigma_{\alpha}}{B}\left[\frac{\mathrm{d} \Pi_{i j}^{(0)}}{\mathrm{d} t}+\Pi_{i j}^{(0)} \frac{\partial u_{k}}{\partial x_{k}}+\Pi_{i k}^{(0)} \frac{\partial u_{j}}{\partial x_{k}}+\Pi_{j k}^{(0)} \frac{\partial u_{i}}{\partial x_{k}}+\frac{\partial Q_{i j k}^{(0)}}{\partial x_{k}}\right] .
$$

Before proceeding in the actual solution of the above equations, let us comment on their symmetry properties, in particular with respect to the magnetic-field direction.

\section{B.2. Symmetry considerations on the perturbed equations}

Let us consider the three operators, $\mathcal{L}_{\boldsymbol{B}}, \mathcal{R}_{\boldsymbol{u}}$ and $\mathcal{D}$. If we invert the direction of the magnetic field, $\boldsymbol{B} \rightarrow-\boldsymbol{B}$, then such operators transform as

$$
\begin{aligned}
& \mathcal{L}_{B}[\bullet] \quad \rightarrow \quad \mathcal{L}_{-B}[\bullet]=-\mathcal{L}_{B}[\bullet] \\
& \mathcal{R}_{u}[\bullet] \quad \rightarrow \quad \mathcal{R}_{u}[\bullet] \\
& \mathcal{D}[\bullet] \quad \rightarrow \quad \mathcal{D}[\bullet],
\end{aligned}
$$

and this symmetry property has a direct consequence on the solutions.

Let us consider the zeroth-order equation, (B 9), and a possible solution $\Pi_{+}^{(0)}$. Then, if we reverse the direction of the magnetic field, the linear operator $\mathcal{L}_{\boldsymbol{B}}$ also changes sign, but the zeroth-order equation remains the same and $\boldsymbol{\Pi}_{+}^{(0)}$ is still a solution (i.e. if $\boldsymbol{\Pi}_{-}^{(0)}$ is the solution when the magnetic-field direction is reversed, then $\boldsymbol{\Pi}_{-}^{(0)}=\boldsymbol{\Pi}_{+}^{(0)}$ must hold in order to have a unique solution). Therefore, $\boldsymbol{\Pi}^{(0)}$ is invariant under magnetic-field inversion and we can drop the ' + ' and ' - ' subscripts (see § B.3).

Let $\boldsymbol{\Pi}_{+}^{(1)}$ be a solution of the first-order equation (B 10),

$$
\mathcal{L}_{\boldsymbol{B}}\left[\boldsymbol{\Pi}_{+}^{(1)}\right]=\mathcal{R}_{\boldsymbol{u}}\left[\boldsymbol{\Pi}^{(0)}\right]+\mathcal{D}\left[\boldsymbol{Q}^{(0)}\right] .
$$

Now consider the same configuration, but with just the magnetic field in the opposite direction, i.e. $\boldsymbol{b} \rightarrow-\boldsymbol{b}$. Regardless of the actual behaviour of the gyrotropic heat-flux tensor, $\boldsymbol{Q}^{(0)}$, with respect to such inversion ${ }^{11}$, if we assume that the first-order solution $\boldsymbol{\Pi}_{+}^{(1)}$ is invariant with respect to $\boldsymbol{b} \rightarrow-\boldsymbol{b}$, we then obtain a different equation:

$$
\mathcal{L}_{\boldsymbol{B}}\left[\boldsymbol{\Pi}_{+}^{(1)}\right]=-\mathcal{R}_{u}\left[\boldsymbol{\Pi}^{(0)}\right] \mp \mathcal{D}\left[\boldsymbol{Q}^{(0)}\right],
$$

where the $\mp$ sign in front of $\mathcal{D}\left[\boldsymbol{Q}^{(0)}\right]$ takes into account for any possible behaviour of $\boldsymbol{Q}^{(0)}$ with respect to such an inversion. Let us drop the heat-flux contribution for a moment and consider the two equations, $\mathcal{L}_{\boldsymbol{B}}\left[\boldsymbol{\Pi}_{+}^{(1)}\right]=\mathcal{R}_{\boldsymbol{u}}\left[\boldsymbol{\Pi}^{(0)}\right]$ and $\mathcal{L}_{\boldsymbol{B}}\left[\boldsymbol{\Pi}_{+}^{(1)}\right]=$ $-\mathcal{R}_{u}\left[\boldsymbol{\Pi}^{(0)}\right]$. Clearly, a non-zero solution $\boldsymbol{\Pi}_{+}^{(1)}$ cannot satisfy simultaneously the two equations above, and so we must admit that there exists a different solution, $\boldsymbol{\Pi}_{-}^{(1)}$. Due to the linear nature of the operators, it is immediate to see that a relation $\boldsymbol{\Pi}_{-}^{(1)}=-\boldsymbol{\Pi}_{+}^{(1)}$ must hold. With the contribution of the heat flux the relation might not be straightforward as $\boldsymbol{\Pi}_{-}^{(1)}=-\boldsymbol{\Pi}_{+}^{(1)}$, but, again, being $\mathcal{L}_{\boldsymbol{B}}, \mathcal{R}_{\boldsymbol{u}}$ and $\mathcal{D}$ linear operators, there will be anyway a part of $\boldsymbol{\Pi}^{(1)}$ that changes sign when $\boldsymbol{b} \rightarrow-\boldsymbol{b}$. This is a feature deeply encoded in the governing equations of a plasma, but it first emerges only when the fluid hierarchy is retained up to the pressure-tensor equation (Cerri et al. 2014; Del Sarto et al. 2016) or first-order FLR corrections are included (Hazeltine, Kotschenreuther \& Morrison 1985; Hsu, Hazeltine \& Morrison 1986; Ramos 2005b; Cerri et al. 2013).

\footnotetext{
${ }^{11}$ One can show that $\boldsymbol{Q}^{(0)}$ has to be a solution of $\mathcal{L}_{\boldsymbol{B}}\left[\boldsymbol{Q}^{(0)}\right]=0$ and it will therefore be a combination of the type $\boldsymbol{Q}^{(0)}=q_{\|} \boldsymbol{b} \boldsymbol{b} \boldsymbol{b}+q_{\perp}\{\boldsymbol{\tau} \boldsymbol{b}\}^{(\mathrm{sym})}$ (Goswami, Passot \& Sulem 2005). This means that the gyrotropic heat-flux tensor changes sign when $\boldsymbol{b} \rightarrow-\boldsymbol{b}$. However, this does not play a role in the following argument.
} 


\section{B.3. Zeroth-order solution: gyrotopic pressure tensor}

At zero order, $\boldsymbol{\Pi}^{(0)}$ must satisfy $\mathcal{L}_{\boldsymbol{B}}\left[\boldsymbol{\Pi}^{(0)}\right]=0$, i.e. it will be a linear combination of the basis vector spanning the kernel of the (self-adjoint) linear operator $\mathcal{L}_{B}$. Any linear combination of the identity, $I$, and of the projector along the magnetic-field direction, $\boldsymbol{b} \boldsymbol{b}$, i.e. $\boldsymbol{\Pi}^{(0)}=p_{1} \boldsymbol{I}+p_{2} \boldsymbol{b} \boldsymbol{b}$, is a zeroth-order solution. Defining the parallel and perpendicular pressures as $p_{\perp}=p_{1}$ and $p_{\|}=p_{1}+p_{2}$, we recover the gyrotropic Chew-Goldberger-Low (CGL) pressure tensor (Chew et al. 1956):

$$
\boldsymbol{\Pi}^{(0)}=p_{\perp} \boldsymbol{\tau}+p_{\|} \boldsymbol{b} \boldsymbol{b} .
$$

The zeroth-order solution is insensitive to the operation $\boldsymbol{b} \rightarrow-\boldsymbol{b}$, as anticipated. Note that the equation for $n=0$, and thus its solution $\boldsymbol{\Pi}_{\alpha}^{(0)}$, does not depend on the velocity field $\boldsymbol{u}$ or on the heat-flux tensor $\boldsymbol{Q}$, so the only information that we need is the direction of the magnetic field, $\boldsymbol{b}$. Finally, note that there is an interesting consequence of this solution in an ordering for which $\omega / \Omega_{c \alpha} \ll 1$ : because the gyrofrequency is inversely proportional to the species' mass, $\Omega_{c \alpha} \propto 1 / m_{\alpha}$, within a low-frequency dynamics we expect the lighter species (e.g. the electrons) to be naturally found very close to a gyrotropic state ${ }^{12}$.

\section{B.4. First-order solution: FLR corrections and dynamic equations for $p_{\|}$and $p_{\perp}$}

Before proceeding in the solution of the first-order equation in the perturbative expansion, (B 10), we recast it in a form that is invariant under the operation $\boldsymbol{b} \rightarrow-\boldsymbol{b}$. In this way, we solve it only once for a solution $\boldsymbol{\Pi}^{(1)}$ that encodes both $\boldsymbol{\Pi}_{+}^{(1)}$ and $\boldsymbol{\Pi}_{-}^{(1)}$. At this stage, we need to take into account the fact that $\boldsymbol{Q}^{(0)}$ changes sign when we reverse the direction of $\boldsymbol{B}$ (see e.g. Goswami et al. 2005). Therefore, we introduce a coefficient that takes into account the relative orientation of the magnetic field with respect to the coordinate axes, $s_{m} \equiv \operatorname{sign}\left[\boldsymbol{b} \cdot \boldsymbol{e}_{m}\right]=\operatorname{sign}\left[b_{m}\right]\left(\operatorname{such}\right.$ that $s_{m}^{-1}=s_{m}$ ), where $\boldsymbol{e}_{m}$ is the unit vector along the $m$-axis of the reference system. The invariant equation now reads (Cerri et al. 2013)

$$
\begin{aligned}
& \left(\epsilon_{i l m} \Pi_{l j}^{(1)}+\epsilon_{j l m} \Pi_{l i}^{(1)}\right) b_{m} \\
& \quad=\frac{s_{m} \sigma_{\alpha}}{B}\left[\frac{\mathrm{d} \Pi_{i j}^{(0)}}{\mathrm{d} t}+\Pi_{i j}^{(0)} \frac{\partial u_{k}}{\partial x_{k}}+\Pi_{i k}^{(0)} \frac{\partial u_{j}}{\partial x_{k}}+\Pi_{j k}^{(0)} \frac{\partial u_{i}}{\partial x_{k}}\right]+\frac{\sigma_{\alpha}}{B} \frac{\partial Q_{i j k}^{(0)}}{\partial x_{k}} .
\end{aligned}
$$

By evaluating every term in the above equation (see, e.g. Cerri et al. 2013), one eventually gets the dynamic equations for the zeroth-order pressure components,

$$
\begin{aligned}
& \frac{\partial p_{\| \alpha}}{\partial t}+\nabla \cdot\left(p_{\| \alpha} \boldsymbol{u}_{\alpha}\right)+2 p_{\| \alpha}\left(\boldsymbol{b} \boldsymbol{b}: \nabla \boldsymbol{u}_{\alpha}\right)+\nabla \cdot\left(q_{\| \alpha} \boldsymbol{b}\right)-2 q_{\perp \alpha}(\nabla \cdot \boldsymbol{b})=0, \\
& \frac{\partial p_{\perp \alpha}}{\partial t}+\nabla \cdot\left(p_{\perp \alpha} \boldsymbol{u}_{\alpha}\right)+p_{\perp \alpha}\left(\boldsymbol{\tau}: \nabla \boldsymbol{u}_{\alpha}\right)+\nabla \cdot\left(q_{\perp \alpha} \boldsymbol{b}\right)+q_{\perp \alpha}(\nabla \cdot \boldsymbol{b})=0
\end{aligned}
$$

and the expressions for the components of $\boldsymbol{\Pi}_{\alpha}^{(1)}$,

$$
\Pi_{\alpha, x x}^{(1)}=-\Pi_{\alpha, y y}^{(1)}=-\frac{s_{3} \sigma_{\alpha}}{2} \frac{p_{\perp \alpha}}{B}\left(\frac{\partial u_{\alpha, x}}{\partial y}+\frac{\partial u_{\alpha, y}}{\partial x}\right)
$$

\footnotetext{
${ }^{12}$ This might not be true everywhere, e.g. if processes such as reconnection are involved (see, e.g. Scudder \& Daughton 2008; Aunai et al. 2013).
} 


$$
\begin{gathered}
\Pi_{\alpha, x y}^{(1)}=\Pi_{\alpha, y x}^{(1)}=-\frac{s_{3} \sigma_{\alpha}}{2} \frac{p_{\perp \alpha}}{B}\left(\frac{\partial u_{\alpha, y}}{\partial y}-\frac{\partial u_{\alpha, x}}{\partial x}\right) \\
\Pi_{\alpha, x z}^{(1)}=\Pi_{\alpha, z x}^{(1)}=-\frac{s_{3} \sigma_{\alpha}}{B}\left[\left(2 p_{\| \alpha}-p_{\perp \alpha}\right) \frac{\partial u_{\alpha, y}}{\partial z}+p_{\perp \alpha} \frac{\partial u_{\alpha, z}}{\partial y}\right]-\frac{\sigma_{\alpha}}{B} \frac{\partial q_{\perp \alpha}}{\partial y} \\
\Pi_{\alpha, y z}^{(1)}=\Pi_{\alpha, z y}^{(1)}=\frac{s_{3} \sigma_{\alpha}}{B}\left[\left(2 p_{\| \alpha}-p_{\perp \alpha}\right) \frac{\partial u_{\alpha, x}}{\partial z}+p_{\perp \alpha} \frac{\partial u_{\alpha, z}}{\partial x}\right]+\frac{\sigma_{\alpha}}{B} \frac{\partial q_{\perp \alpha}}{\partial x} \\
\Pi_{\alpha, z z}^{(1)}=0 .
\end{gathered}
$$

By neglecting the parallel heat fluxes, $q_{\|}$and $q_{\perp}$, the above expressions can be compared with the classical results given in Braginskii (1965) for the collisional case by setting $\eta_{0}=\eta_{1}=\eta_{2}=0, \eta_{3}=p_{\perp} / 2 \Omega$ and $\eta_{4}=p_{\perp} / \Omega$ in the Braginskii's gyro-viscous coefficients. Moreover, in our expressions there is a contribution to $\Pi_{\alpha, x z}^{(1)}$ and to $\Pi_{\alpha, y z}^{(1)}$ that is due to the pressure anisotropy, $\left[2\left(p_{\|}-p_{\perp}\right) / \Omega\right] \partial_{z} u_{\alpha, x}$ and $\left[2\left(p_{\|}-p_{\perp}\right) / \Omega\right] \partial_{z} u_{\alpha, y}$, respectively, which is missing in Braginskii (1965) because of the assumed isotropic temperature, $T_{\|}=T_{\perp}=T$. The above expressions for the FLR corrections explicitly account for the orientation of the magnetic field with respect to the $z$-axis through the $s_{3}$ coefficient.

\section{Appendix C. Convergence of the FLR expansion to the full pressure tensor}

We expand the pressure tensor for the species $\alpha, \boldsymbol{\Pi}_{\alpha}$, as a power series in the small parameter $\varepsilon_{\alpha} \equiv \rho_{\alpha} / L \ll 1$ :

$$
\boldsymbol{\Pi}_{\alpha}=\sum_{n=0}^{\infty} \varepsilon_{\alpha}^{n} \boldsymbol{\Pi}_{\alpha}^{(n)}
$$

and we perform an equivalent expansion for the heat-flux tensor, $\boldsymbol{Q}_{\alpha}$. Within the $e \mathrm{TF}$ ordering (Cerri et al. 2013), the dimensionless $n$ th-order pressure-tensor equation reads

$$
\mathcal{L}_{B}\left[\Pi_{\alpha, i j}^{(n)}\right]=\hat{\mathcal{R}}_{u}\left[\Pi_{\alpha, i j}^{(n-1)}\right]+\mathcal{D}\left[Q_{\alpha, i j(k)}^{(n-1)}\right],
$$

where

$$
\begin{gathered}
\mathcal{L}_{B}\left[\Pi_{\alpha, i j}^{(n)}\right] \equiv\left(\epsilon_{i l m} \Pi_{\alpha, l j}^{(n)}+\epsilon_{j l m} \Pi_{\alpha, l i}^{(n)}\right) B_{m}, \\
\hat{\mathcal{R}}_{u}\left[\Pi_{\alpha, i j}^{(n-1)}\right] \equiv s_{m} \sigma_{\alpha}\left[\frac{\mathrm{d} \Pi_{\alpha, i j}^{(n-1)}}{\mathrm{d} t}+\Pi_{\alpha, i j}^{(n-1)} \frac{\partial u_{\alpha, k}}{\partial x_{k}}+\Pi_{\alpha, i k}^{(n-1)} \frac{\partial u_{\alpha, j}}{\partial x_{k}}+\Pi_{\alpha, j k}^{(n-1)} \frac{\partial u_{\alpha, i}}{\partial x_{k}}\right], \\
\mathcal{D}\left[Q_{\alpha, i j(k)}^{(n-1)}\right] \equiv \sigma_{\alpha} \frac{\partial Q_{\alpha, j i k}^{(n-1)}}{\partial x_{k}},
\end{gathered}
$$

where $\epsilon_{i j k}$ is the Levi-Civita symbol, $\sigma_{\alpha} \equiv \operatorname{sign}\left(e_{\alpha}\right)$ is the sign of the electric charge of the $\alpha$ species and $s_{m} \equiv \operatorname{sign}\left(\boldsymbol{b} \cdot \boldsymbol{e}_{m}\right)$ is the relative orientation of the magnetic field with respect to the $m$-axis of the reference system $\left(\boldsymbol{b} \equiv \boldsymbol{B} /|\boldsymbol{B}|\right.$ and $\boldsymbol{e}_{m}$ are the unit vectors along the magnetic field and along the $m$-axis, respectively). We want to find an exact solution for $\boldsymbol{\Pi}_{\alpha}$, i.e. a convergent series as in (C 1) that solves (C2) for all $n$.

First of all, we note that for $n=0$, the solution of (C2), which reduces to $\mathcal{L}_{B}\left[\Pi_{\alpha, i j}^{(0)}\right]=0$, is the gyrotropic CGL pressure tensor (Chew et al. 1956):

$$
\boldsymbol{\Pi}_{\alpha}^{(0)}=p_{\perp \alpha} \boldsymbol{\tau}+p_{\| \alpha} \boldsymbol{b} \boldsymbol{b},
$$

where $\boldsymbol{\tau} \equiv \boldsymbol{I}-\boldsymbol{b} \boldsymbol{b}$ is the projector onto the plane perpendicular to the magnetic field. 


\section{C.1. Assumptions and general nth-order solution}

In order to find a solution of (C 2) to all orders, we first need to make four assumption on the configuration, on the energy and on the closure. The first is to (i) neglect the heat-flux tensor. The second is that (ii) the inhomogeneity direction, the flow direction and the magnetic-field direction form a right-handed basis ${ }^{13}$, e.g. $\boldsymbol{u}=u_{y}(x) \boldsymbol{e}_{y}$ and $\boldsymbol{B}=$ $B_{z}(x) \boldsymbol{e}_{z}$. The third assumption is (iii) stationarity, i.e. no time dependence. Finally, (iv) we assume that any contribution to the pressure tensor beyond the gyrotropic pressure is traceless, which means that we are considering corrections at constant thermal energy. So, summarizing the hypothesis under which we find the solution:

(i) $\boldsymbol{Q}^{(n)}=0 \forall n$;

(ii) $\boldsymbol{\nabla} \cdot \boldsymbol{u}=0$ and $\boldsymbol{B} \times(\boldsymbol{\nabla} \times \boldsymbol{u})=0$;

(iii) $\partial / \partial t=0$;

(iv) $\operatorname{Tr}\left[\boldsymbol{\Pi}_{\alpha}^{(n)}\right]=0 \forall n \geqslant 1$.

Under the assumptions (i)-(iv), considering the inhomogeneity to be in $x$-direction for simplicity, the solution of (C2) $\forall n \geqslant 1$ is:

$$
\left.\begin{array}{c}
\Pi_{\alpha, i j}^{(n)}=0 \quad \text { if } i \neq j, \\
\Pi_{\alpha, x x}^{(n)}=-\Pi_{\alpha, y y}^{(n)}=\left(\widetilde{\chi}_{\alpha}(x)\right)^{n} p_{\perp \alpha}, \\
\Pi_{\alpha, z z}^{(n)}=0,
\end{array}\right\}
$$

where we have defined the function $\widetilde{\chi}_{\alpha}(x)$ as

$$
\widetilde{\chi}_{\alpha}(x) \equiv-\sigma_{\alpha} \frac{\boldsymbol{\omega}_{\alpha} \cdot \boldsymbol{b}}{2 \Omega_{c \alpha}}=-\sigma_{\alpha} \frac{s_{z}}{2|\boldsymbol{B}|} \frac{\mathrm{d} u_{\alpha, y}}{\mathrm{~d} x} .
$$

Note that, in general, $\Pi_{z z}^{(n)}$ is undetermined at each order, so we make the reasonable choice to take it non-zero only for $n=0$, i.e. $\Pi_{z z}^{(n)}=p_{\|} \delta_{n 0}$, which then, together with the traceless condition (iv), gives us the relation $\Pi_{x x}^{(n)}+\Pi_{y y}^{(n)}=2 p_{\perp} \delta_{n 0}$.

\section{C.2. General nth-order solution: proof}

We now proceed to prove that (C 5) is the solution of (C 2), for all $n$. In order to do that, we are going to use the so-called mathematical induction method. Later on, we will omit the $\alpha$ index for the species for shortness.

(i) $n=1$ : For $n=1$, (C 2) is $\mathcal{L}_{B}\left[\Pi_{\alpha, i j}^{(1)}\right]=\hat{\mathcal{R}}_{u}\left[\Pi_{\alpha, i j}^{(0)}\right]$, or written in matrix form

$$
\left(\begin{array}{ccc}
2 \Pi_{x y}^{(1)} & \Pi_{y y}^{(1)}-\Pi_{x x}^{(1)} & \Pi_{y z}^{(1)} \\
\Pi_{y y}^{(1)}-\Pi_{x x}^{(1)} & -2 \Pi_{x y}^{(1)} & -\Pi_{x z}^{(1)} \\
\Pi_{y z}^{(1)} & -\Pi_{x z}^{(1)} & 0
\end{array}\right)=\frac{s_{3} \sigma}{|\boldsymbol{B}|}\left(\begin{array}{ccc}
\frac{\mathrm{d}}{\mathrm{d} t} \Pi_{x x}^{(0)} & \Pi_{x x}^{(0)} \frac{\mathrm{d} u_{y}}{\mathrm{~d} x} & 0 \\
\Pi_{x x}^{(0)} \frac{\mathrm{d} u_{y}}{\mathrm{~d} x} & -\frac{\mathrm{d}}{\mathrm{d} t} \Pi_{x x}^{(0)} & 0 \\
0 & 0 & 0
\end{array}\right)
$$

whose solution under our assumptions is:

$$
\left.\begin{array}{c}
\Pi_{\alpha, i j}^{(1)}=0 \quad \text { if } i \neq j \\
\Pi_{\alpha, x x}^{(1)}=-\Pi_{\alpha, y y}^{(1)}=-\frac{s_{z} \sigma}{2|\boldsymbol{B}|} \frac{\mathrm{d} u_{y}}{\mathrm{~d} x} p_{\perp} \equiv \widetilde{\chi}(x) p_{\perp} \\
\Pi_{\alpha, z z}^{(1)}=0
\end{array}\right\}
$$

\footnotetext{
${ }^{13}$ Note that for incompressible flows, $\boldsymbol{\nabla} \cdot \boldsymbol{u}=0$, this condition corresponds to the case $\boldsymbol{B} \times \boldsymbol{\omega}=\boldsymbol{B} \times(\boldsymbol{\nabla} \times \boldsymbol{u})=0$ that has been considered by Del Sarto \& Pegoraro (2018).
} 
where we have used the assumptions (ii) and (iii) in order to have $\mathrm{d} \Pi_{x x}^{(0)} / \mathrm{d} t=$ 0 : since every quantity can be function only of $x$ and the flow is along the $y$-direction due to assumption (ii), we get $\boldsymbol{u} \cdot \nabla \Pi_{x x}^{(0)}=u_{y} \partial \Pi_{x x}^{(0)} / \partial y=0$ and thus, due also to the stationarity assumption (iii), $(\mathrm{d} / \mathrm{d} t) \Pi_{x x}^{(0)}=\left(\partial / \partial t+u_{y} \partial / \partial y\right) \Pi_{x x}^{(0)}=0$.

(ii) $n=2$ : For $n=2$, (C 2) is $\mathcal{L}_{\boldsymbol{B}}\left[\Pi_{\alpha, i j}^{(2)}\right]=\hat{\mathcal{R}}_{u}\left[\Pi_{\alpha, i j}^{(1)}\right]$, with $\Pi_{\alpha, i j}^{(1)}$ given in (C 8). Such an equation, written in matrix form reads

$$
\left(\begin{array}{ccc}
2 \Pi_{x y}^{(2)} & \Pi_{y y}^{(2)}-\Pi_{x x}^{(2)} & \Pi_{y z}^{(2)} \\
\Pi_{y y}^{(2)}-\Pi_{x x}^{(2)} & -2 \Pi_{x y}^{(2)} & -\Pi_{x z}^{(2)} \\
\Pi_{y z}^{(2)} & -\Pi_{x z}^{(2)} & 0
\end{array}\right)=\frac{s_{z} \sigma}{|\boldsymbol{B}|}\left(\begin{array}{ccc}
\frac{\mathrm{d}}{\mathrm{d} t} \Pi_{x x}^{(1)} & \Pi_{x x}^{(1)} \frac{\mathrm{d} u_{y}}{\mathrm{~d} x} & 0 \\
\Pi_{x x}^{(1)} \frac{\mathrm{d} u_{y}}{\mathrm{~d} x} & -\frac{\mathrm{d}}{\mathrm{d} t} \Pi_{x x}^{(1)} & 0 \\
0 & 0 & 0
\end{array}\right)
$$

whose solution, using again the fact that $\mathrm{d} \Pi_{x x}^{(1)} / \mathrm{d} t=0$, is:

$$
\left.\begin{array}{c}
\Pi_{\alpha, i j}^{(2)}=0 \quad \text { if } i \neq j \\
\Pi_{\alpha, x x}^{(2)}=-\Pi_{\alpha, y y}^{(2)}=\frac{1}{4|\boldsymbol{B}|^{2}}\left(\frac{\mathrm{d} u_{y}}{\mathrm{~d} x}\right)^{2} p_{\perp} \equiv(\widetilde{\chi}(x))^{2} p_{\perp} \\
\Pi_{\alpha, z z}^{(2)}=0
\end{array}\right\}
$$

where we used the fact that $s_{z}^{2}=1$ and $\sigma^{2}=1$.

(iii) Inductive step: We now assume that (C 5) is the correct $n$ th-order solution and we want to solve $(\mathrm{C} 2)$ for the $(n+1)$ th order. That is, $\mathcal{L}_{B}\left[\Pi_{\alpha, i j}^{(n+1)}\right]=\hat{\mathcal{R}}_{u}\left[\Pi_{\alpha, i j}^{(n)}\right]$, which in matrix form reads

$$
\left(\begin{array}{ccc}
2 \Pi_{x y}^{(n+1)} & \Pi_{y y}^{(n+1)}-\Pi_{x x}^{(n+1)} & \Pi_{y z}^{(n+1)} \\
\Pi_{y y}^{(n+1)}-\Pi_{x x}^{(n+1)} & -2 \Pi_{x y}^{(n+1)} & -\Pi_{x z}^{(n+1)} \\
\Pi_{y z}^{(n+1)} & -\Pi_{x z}^{(n+1)} & 0
\end{array}\right)=\frac{s_{z} \sigma}{|\boldsymbol{B}|}\left(\begin{array}{ccc}
\frac{\mathrm{d}}{\mathrm{d} t} \Pi_{x x}^{(n)} & \Pi_{x x}^{(n)} \frac{\mathrm{d} u_{y}}{\mathrm{~d} x} & 0 \\
\Pi_{x x}^{(n)} \frac{\mathrm{d} u_{y}}{\mathrm{~d} x} & -\frac{\mathrm{d}}{\mathrm{d} t} \Pi_{x x}^{(n)} & 0 \\
0 & 0 & 0
\end{array}\right)
$$

whose solution, using again the fact that our assumptions are such that $\mathrm{d} \Pi_{x x}^{(n)} / \mathrm{d} t=$ 0 , is:

$$
\left.\begin{array}{c}
\Pi_{\alpha, i j}^{(n+1)}=0 \quad \text { if } i \neq j, \\
\Pi_{\alpha, x x}^{(n+1)}=-\Pi_{\alpha, y y}^{(n+1)}=\left(-\frac{s_{z} \sigma}{2|\boldsymbol{B}|} \frac{\mathrm{d} u_{y}}{\mathrm{~d} x}\right)^{n+1} p_{\perp} \equiv(\widetilde{\chi}(x))^{n+1} p_{\perp} \\
\Pi_{\alpha, z z}^{(n+1)}=0
\end{array}\right\}
$$

which finally proves the thesis.

\section{C.3. Summability, convergence and stability of the complete pressure tensor}

Now that we have proved the expression for the general $n$ th-order solution of (C2), we want to go back from the FLR expansion to the full pressure tensor, (C 1). In order to be able to do that, the series must be summable and it should converge. 
If we put all the FLR contributions together, the full pressure-tensor components are:

$$
\begin{gathered}
\Pi_{i j}=0 \quad \text { if } i \neq j, \\
\Pi_{z z}=p_{\|}, \\
\Pi_{x x}=\left[1+\widetilde{\chi}+\widetilde{\chi}^{2}+\cdots\right] p_{\perp}=\left[1+\tilde{\chi} \sum_{n=0}^{\infty}(\widetilde{\chi})^{n}\right] p_{\perp}, \\
\Pi_{y y}=\left[1-\tilde{\chi}-\widetilde{\chi}^{2}-\cdots\right] p_{\perp}=\left[1-\tilde{\chi} \sum_{n=0}^{\infty}(\widetilde{\chi})^{n}\right] p_{\perp},
\end{gathered}
$$

so the main request for absolute convergence is that the geometric series $\sum_{n}|\widetilde{\chi}|^{n}$ converge, which is true if and only if

$$
|\widetilde{\chi}(x)|<1 \Longleftrightarrow|\boldsymbol{\omega} \cdot \boldsymbol{b}|<2 \Omega_{c} \quad \forall x,
$$

which is the absolute convergence condition from the mathematical point of view and represents a limit on the shear strength. If the condition (C 17) holds, then the resulting diagonal components of the pressure tensor are

$$
\begin{gathered}
\Pi_{x x}=\left(1+\frac{\widetilde{\chi}(x)}{1-\widetilde{\chi}(x)}\right) p_{\perp}, \\
\Pi_{y y}=\left(1-\frac{\widetilde{\chi}(x)}{1-\widetilde{\chi}(x)}\right) p_{\perp}, \\
\Pi_{z z}=p_{\|} .
\end{gathered}
$$

However, since the components of the (diagonal) pressure tensor cannot be negative in order to have a physical meaning, the function $\widetilde{\chi}(x)$ - and thus the shear strength $\mathrm{d} u_{y} / \mathrm{d} x-$ has to fulfil the positivity condition. This request gives a physical condition on the shear strength which reads

$$
\widetilde{\chi}(x) \leqslant \frac{1}{2} \Longleftrightarrow \omega \cdot b \geqslant-\Omega_{c},
$$

where now, in principle, the shear can be as negative as one wishes, without no limitations. If we put together the physical condition (C21) and the mathematical condition (C17), gives the asymmetric condition

$$
-1<\widetilde{\chi}(x) \leqslant \frac{1}{2} \quad \Longleftrightarrow \quad-\Omega_{c} \leqslant \omega \cdot \boldsymbol{b}<2 \Omega_{c} \quad \forall x,
$$

The condition above is also a stability condition for the shear-flow configuration. In fact, that is in agreement with Del Sarto et al. (2016), where the stability condition is found to be $\Omega^{\prime} \equiv \Omega+\partial_{x} u_{y} \geqslant 0$, which translated in our notation correspond to $\widetilde{\chi}(x) \leqslant 1 / 2^{14}$.

\footnotetext{
${ }^{14}$ Note that the condition $\tilde{\chi}>-1$ in (C 22) originates from the fact that we are requiring that the pressure tensor $\Pi$ can be expanded in an infinite series of a small parameter $\varepsilon$, (C1), and that the resulting contributions $\Pi^{(n)}$ should converge again to $\Pi$ when 'summed back'. However those assumptions are not made when dealing with the pressure-tensor equation, so the upper bound $\boldsymbol{\omega} \cdot \boldsymbol{b}<2 \Omega_{c}$ does enter the full pressure-tensor case (see Cerri et al. 2014).
} 


\section{REFERENCES}

ALFVÉn, H. 1942 Existence of electromagnetic-hydrodynamic waves. Nature 150, 405-406.

Attico, N. \& Pegoraro, F. 1999 Periodic equilibria of the Vlasov-Maxwell system. Phys. Plasmas 6, 767-770.

Aunai, N., Hesse, M. \& KuZnetsova, M. 2013 Electron nongyrotropy in the context of collisionless magnetic reconnection. Phys. Plasmas 20 (9), 092903.

BisKamP, D. 2008 Magnetohydrodynamic Turbulence Cambridge University Press.

Bobrova, N. A., Bulanov, S. V., Sakai, J. I. \& SugiYama, D. 2001 Force-free equilibria and reconnection of the magnetic field lines in collisionless plasma configurations. Phys. Plasmas 8, 759-768.

BraginskiI, S. I. 1965 Transport processes in a plasma. Rev. Plasma Phys. 1, 205.

Brizard, A. J. \& HAHM, T. S. 2007 Foundations of nonlinear gyrokinetic theory. Rev. Mod. Phys. 79, 421-468.

Cai, D., Storey, L. R. O. \& Neubert, T. 1990 Kinetic equilibria of plasma shear layers. Phys. Fluids B 2, 75-85.

Camporeale, E., Sorriso-Valvo, L., Califano, F. \& Retinò, A. 2018 Coherent structures and spectral energy transfer in turbulent plasma: a space-filter approach. Phys. Rev. Lett. 120 (12), 125101.

Cassak, P. A., Baylor, R. N., Fermo, R. L., Beidler, M. T., Shay, M. A., Swisdak, M., DRAKe, J. F. \& KARIMABADI, H. 2015 Fast magnetic reconnection due to anisotropic electron pressure. Phys. Plasmas 22 (2), 020705.

CERRI, S. S. 2012 Fluid modeling of kinetic effects in collisionless magnetized plasmas and application to solar wind-magnetosphere interaction. Master's thesis, University of Pisa, Italy.

Cerri, S. S. \& Califano, F. 2017 Reconnection and small-scale fields in 2D-3V hybrid-kinetic driven turbulence simulations. New J. Phys. 19 (2), 025007.

Cerri, S. S., Franci, L., Califano, F., Landi, S. \& Hellinger, P. 2017 Plasma turbulence at ion scales: a comparison between particle in cell and Eulerian hybrid-kinetic approaches. J. Plasma Phys. 83 (2), 705830202.

Cerri, S. S., Henri, P., Califano, F., Del Sarto, D., Faganello, M. \& Pegoraro, F. 2013 Extended fluid models: pressure tensor effects and equilibria. Phys. Plasmas 20 (11), 112112.

Cerri, S. S., KunZ, M. W. \& Califano, F. 2018 Dual phase-space cascades in 3D hybrid-VlasovMaxwell turbulence. Astrophys. J. Lett. 856, L13.

Cerri, S. S., Pegoraro, F., Califano, F., Del Sarto, D. \& Jenko, F. 2014 Pressure tensor in the presence of velocity shear: stationary solutions and self-consistent equilibria. Phys. Plasmas 21 (11), 112109.

ChANDRASEKHAR, S. 1956 On the stability of the simplest solution of the equations of hydromagnetics. Proc. Natl Acad. Sci. 42, 273-276.

Chapman, S. \& Ferraro, V. C. A. 1930 A new theory of magnetic storms. Nature 126, 129-130.

Chew, G. F., Goldberger, M. L. \& Low, F. E. 1956 The Boltzmann equation and the one-fluid hydromagnetic equations in the absence of particle collisions. Proc. R. Soc. Lond. A 236, $112-118$.

De Camillis, S., Cerri, S. S., Califano, F. \& Pegoraro, F. 2016 Pressure anisotropy generation in a magnetized plasma configuration with a shear flow velocity. Plasma Phys. Control. Fusion 58, 045007.

Del Sarto, D. \& Pegoraro, F. 2018 Shear-induced pressure anisotropization and correlation with fluid vorticity in a low collisionality plasma. Mon. Not. R. Astron. Soc. 475, 181-192.

Del Sarto, D., Pegoraro, F. \& Califano, F. 2016 Pressure anisotropy and small spatial scales induced by velocity shear. Phys. Rev. E 93 (5), 053203.

Del Sarto, D., Pegoraro, F. \& Tenerani, A. 2017 'Magneto-elastic' waves in an anisotropic magnetised plasma. Plasma Phys. Control. Fusion 59, 045002.

Delamere, P. A., Wilson, R. J., Eriksson, S. \& Bagenal, F. 2013 Magnetic signatures of Kelvin-Helmholtz vortices on Saturn's magnetopause: global survey. J. Geophys. Res. (Space Phys.) 118, 393-404. 
Dmitriev, A. V. \& Suvorova, A. V. 2012 Traveling magnetopause distortion related to a large-scale magnetosheath plasma jet: THEMIS and ground-based observations. J. Geophys. Res. (Space Phys.) 117, A08217.

Dong, C., Lingam, M., MA, Y. \& Cohen, O. 2017 Is proxima centauri b habitable? A study of atmospheric loss. Astrophys. J. Lett. 837, L26.

Fadanelli, S., Faganello, M., Califano, F., Cerri, S. S., Pegoraro, F. \& Lavraud, B. 2018 North-South asymmetric Kelvin-Helmholtz instability and induced reconnection at the Earth's magnetospheric flanks. J. Geophys. Res. ArXiv e-prints (accepted).

Faganello, M. \& CAlifano, F. 2017 Magnetized Kelvin-Helmholtz instability: theory and simulations in the Earth's magnetosphere context. J. Plasma Phys. 83 (6), 535830601.

Faganello, M., Califano, F. \& Pegoraro, F. 2008 Competing mechanisms of plasma transport in inhomogeneous configurations with velocity shear: the solar-wind interaction with earth's magnetosphere. Phys. Rev. Lett. 100 (1), 015001.

Faganello, M., Califano, F., Pegoraro, F. \& Andreussi, T. 2012 Double mid-latitude dynamical reconnection at the magnetopause: an efficient mechanism allowing solar wind to enter the Earth's magnetosphere. Europhys. Lett. 100, 69001.

FERraro, N. M. 2007 Finite larmor radius effects on the magnetorotational instability. Astrophys. J. 662, 512-516.

Ferraro, V. C. A. 1937 The non-uniform rotation of the Sun and its magnetic field. Mon. Not. R. Astron. Soc. 97, 458.

Foullon, C., Farrugia, C. J., Fazakerley, A. N., Owen, C. J., Gratton, F. T. \& Torbert, R. B. 2008 Evolution of Kelvin-Helmholtz activity on the dusk flank magnetopause. J. Geophys. Res. Space Phys. 113, A11203.

Franci, L., Cerri, S. S., Califano, F., Landi, S., Papini, E., Verdini, A., Matteini, L., Jenko, F. \& Hellinger, P. 2017 Magnetic reconnection as a driver for a sub-ion-scale cascade in plasma turbulence. Astrophys. J. Lett. 850, L16.

Franci, L., Hellinger, P., Matteini, L., Verdini, A. \& Landi, S. 2016 Two-dimensional hybrid simulations of kinetic plasma turbulence: current and vorticity versus proton temperature. American Institute of Physics Conference Series, vol. 1720. American Institute of Physics Conference Series, p. 040003.

Fujimoto, M. \& Terasawa, T. 1995 Anomalous ion mixing within an MHD scale Kelvin-Helmholtz vortex. 2: effects of inhomogeneity. J. Geophys. Res. 100, 12.

Gershman, D. J., Raines, J. M., Slavin, J. A., Zurbuchen, T. H., SundberG, T., Boardsen, S. A., Anderson, B. J., Korth, H. \& Solomon, S. C. 2015 MESSENGER observations of multiscale Kelvin-Helmholtz vortices at Mercury. J. Geophys. Res. (Space Phys.) 120, 4354-4368.

Gingell, P. W., Sundberg, T. \& Burgess, D. 2015 The impact of a hot sodium ion population on the growth of the Kelvin-Helmholtz instability in Mercury's magnetotail. J. Geophys. Res. (Space Phys.) 120, 5432-5442.

Goswami, P., Passot, T. \& Sulem, P. L. 2005 A Landau fluid model for warm collisionless plasmas. Phys. Plasmas 12, 102109.

Grad, H. 1960 Reducible problems in magneto-fluid dynamic steady flows. Rev. Mod. Phys. 32, $830-847$.

Greco, A., Valentini, F., Servidio, S. \& Matthaeus, W. H. 2012 Inhomogeneous kinetic effects related to intermittent magnetic discontinuities. Phys. Rev. E 86 (6), 066405.

Groth, C. P. T., De Zeeuw, D. L., Gombosi, T. I. \& Powell, K. G. 2000 Global three-dimensional MHD simulation of a space weather event: CME formation, interplanetary propagation, and interaction with the magnetosphere. J. Geophys. Res. 105, 25053-25078.

Grošelu, D., Cerri, S. S., Bañón Navarro, A., Willmott, C., Told, D., Loureiro, N. F., Califano, F. \& Jenko, F. 2017 Fully kinetic versus reduced-kinetic modeling of collisionless plasma turbulence. Astrophys. J. 847, 28.

Haaland, S., Reistad, J., Tenfjord, P., Gjerloev, J., Maes, L., Dekeyser, J., Maggiolo, R., Anekallu, C. \& Dorville, N. 2014 Characteristics of the flank magnetopause: cluster observations. J. Geophys. Res. (Space Phys.) 119, 9019-9037. 
Hammett, G. W. \& Perkins, F. W. 1990 Fluid moment models for Landau damping with application to the ion-temperature-gradient instability. Phys. Rev. Lett. 64, 3019-3022.

HAU, L.-N. 2002 A note on the energy laws in gyrotropic plasmas. Phys. Plasmas 9, $2455-2457$.

Hau, L.-N., Phan, T.-D., Sonnerup, B. U. O. \& Paschmann, G. 1993 Double-polytropic closure in the magentosheath. Geophys. Res. Lett. 20, 2255-2258.

Hazeltine, R. D., Hsu, C. T. \& Morrison, P. J. 1987 Hamiltonian four-field model for nonlinear tokamak dynamics. Phys. Fluids 30, 3204-3211.

Hazeltine, R. D., Kotschenreuther, M. \& Morrison, P. J. 1985 A four-field model for tokamak plasma dynamics. Phys. Fluids 28, 2466-2477.

Hellinger, P., TrávníčeK, P., Kasper, J. C. \& Lazarus, A. J. 2006 Solar wind proton temperature anisotropy: linear theory and WIND/SWE observations. Geophys. Res. Lett. 33, L09101.

Henri, P., Califano, F., Faganello, M. \& Pegoraro, F. 2012 Magnetised Kelvin-Helmholtz instability in the intermediate regime between subsonic and supersonic regimes. Phys. Plasmas 19 (7), 072908.

Henri, P., Cerri, S. S., Califano, F., Pegoraro, F., Rossi, C., Faganello, M., ŠebeK, O., Trávníček, P. M., Hellinger, P., Frederiksen, J. T. et al. 2013 Nonlinear evolution of the magnetized Kelvin-Helmholtz instability: from fluid to kinetic modeling. Phys. Plasmas 20 (10), 102118.

Hsu, C. T., Hazeltine, R. D. \& Morrison, P. J. 1986 A generalized reduced fluid model with finite ion-gyroradius effects. Phys. Fluids 29, 1480-1487.

HubA, J. D. 1996 The Kelvin-Helmholtz instability: finite Larmor radius magnetohydrodynamics. Geophys. Res. Lett. 23, 2907-2910.

Jia, X., Hansen, K. C., Gombosi, T. I., Kivelson, M. G., Tóth, G., Dezeeuw, D. L. \& RIDLEY, A. J. 2012 Magnetospheric configuration and dynamics of Saturn's magnetosphere: a global MHD simulation. J. Geophys. Res. (Space Phys.) 117, A05225.

Jia, X., Slavin, J. A., Gombosi, T. I., DaldorfF, L. K. S., Tóth, G. \& Van Der Holst, B. 2015 Global mhd simulations of mercury's magnetosphere with coupled planetary interior: induction effect of the planetary conducting core on the global interaction. J. Geophys. Res. (Space Phys.) 120 (6), 4763-4775; 2015JA021143.

Johnson, J. R., Wing, S. \& Delamere, P. A. 2014 Kelvin Helmholtz instability in planetary magnetospheres. Space Sci. Rev. 184, 1-31.

Karimabadi, H., Roytershteyn, V., Daughton, W. \& LiU, Y.-H. $2013 a$ Recent evolution in the theory of magnetic reconnection and its connection with turbulence. Space Sci. Rev. 178, 307-323.

Karimabadi, H., Roytershteyn, V., Wan, M., Matthaeus, W. H., Daughton, W., Wu, P., Shay, M., Loring, B., Borovsky, J., Leonardis, E. et al. $2013 b$ Coherent structures, intermittent turbulence, and dissipation in high-temperature plasmas. Phys. Plasmas 20 (1), 012303 .

KimurA, K. \& Morrison, P. J. 2014 On energy conservation in extended magnetohydrodynamics. Phys. Plasmas 21 (8), 082101.

Kunz, M. W., Stone, J. M. \& QuAtaert, E. 2016 Magnetorotational turbulence and dynamo in a collisionless plasma. Phys. Rev. Lett. 117 (23), 235101.

LANDAU, L. D. 1946 On the vibrations of the electronic plasma. Zh. Eksp. Teor. Fiz. 16, 574.

LAZARIAN, A., EYINK, G. L. \& VishniAC, E. T. 2012 Relation of astrophysical turbulence and magnetic reconnection. Phys. Plasmas 19 (1), 012105.

LERoy, M. H. J. \& KePpens, R. 2017 On the influence of environmental parameters on mixing and reconnection caused by the Kelvin-Helmholtz instability at the magnetopause. Phys. Plasmas 24 (1), 012906.

Liljeblad, E., Sundberg, T., Karlsson, T. \& Kullen, A. 2014 Statistical investigation of Kelvin-Helmholtz waves at the magnetopause of Mercury. J. Geophys. Res. (Space Phys.) 119, 9670-9683.

LiU, Z.-Q., Lu, J. Y., Wang, C., Kabin, K., Zhao, J. S., Wang, M., Han, J. P., Wang, J. Y. \& ZHAO, M. X. 2015 A three-dimensional high mach number asymmetric magnetopause 
model from global mhd simulation. J. Geophys. Res. (Space Phys.) 120 (7), 5645-5666; 2014JA020961.

LÜST, R. \& SCHLÜTER, A. 1954 Kraftfreie Magnetfelder. Zeitschrift für Astrophysik 34, 263.

MACMAHON, A. 1965 Finite gyro-radius corrections to the hydromagnetic equations for a Vlasov plasma. Phys. Fluids 8, 1840-1845.

Mahajan, S. M. \& Hazeltine, R. D. 2000 Sheared-flow generalization of the Harris sheet. Phys. Plasmas 7, 1287-1293.

Malara, F., Pezzi, O. \& Valentini, F. 2018 Exact hybrid Vlasov equilibria for sheared plasmas with in-plane and out-of-plane magnetic field. Phys. Rev. E 97 (5), 053212.

Masters, A., Achilleos, N., Cutler, J. C., Coates, A. J., Dougherty, M. K. \& Jones, G. H. 2012 Surface waves on Saturn's magnetopause. Planet. Space Sci. 65, 109-121.

Matthaeus, W. H. \& Lamkin, S. L. 1986 Turbulent magnetic reconnection. Phys. Fluids 29, 2513-2534.

Merkin, V. G., Lyon, J. G. \& Claudepierre, S. G. 2013 Kelvin-Helmholtz instability of the magnetospheric boundary in a three-dimensional global MHD simulation during northward IMF conditions. J. Geophys. Res. (Space Phys.) 118, 5478-5496.

MiurA, A. 1987 Simulation of Kelvin-Helmholtz instability at the magnetospheric boundary. J. Geophys. Res. 92, 3195-3206.

MıøLhUS, E. 2009 Finite Larmor radius influence on MHD solitary waves. Nonlinear Process. Geophys. 16, 251-264.

NaGAnO, H. 1978 Effect of finite ion Larmor radius on the Kelvin-Helmholtz instability. J. Plasma Phys. 20, 149-160.

Nakamura, T. K. M. \& Fujimoto, M. 2005 Magnetic reconnection within rolled-up MHDscale Kelvin-Helmholtz vortices: two-fluid simulations including finite electron inertial effects. Geophys. Res. Lett. 32, L21102.

Nakamura, T. K. M., Hasegawa, H. \& Shinohara, I. 2010 Kinetic effects on the KelvinHelmholtz instability in ion-to-magnetohydrodynamic scale transverse velocity shear layers: particle simulations. Phys. Plasmas 17 (4), 042119.

NYKYRI, K. \& OTTO, A. 2004 Influence of the Hall term on KH instability and reconnection inside KH vortices. Ann. Geophys. 22, 935-949.

OtTo, A. \& FAIRFIELD, D. H. 2000 Kelvin-Helmholtz instability at the magnetotail boundary: MHD simulation and comparison with Geotail observations. J. Geophys. Res. 105, 21.

Palermo, F., Faganello, M., Califano, F., Pegoraro, F. \& Le Contel, O. 2011 Compressible Kelvin-Helmholtz instability in supermagnetosonic regimes. J. Geophys. Res. (Space Phys.) 116, A04223.

PARAL, J. \& RANKIN, R. 2013 Dawn-dusk asymmetry in the Kelvin-Helmholtz instability at mercury. Nature Commun. 4, 1645.

Parashar, T. N. \& Matthaeus, W. H. 2016 Propinquity of current and vortex structures: effects on collisionless plasma heating. Astrophys. J. 832, 57.

Pezzi, O., Servidio, S., Perrone, D., Valentini, F., Sorriso-Valvo, L., Greco, A., Matthaeus, W. H. \& Veltri, P. 2018 Velocity-space cascade in magnetized plasmas: numerical simulations. Phys. Plasmas 25 (6), 060704; ArXiv e-prints.

Ramos, J. J. $2005 a$ Fluid formalism for collisionless magnetized plasmas. Phys. Plasmas 12, 052102.

Ramos, J. J. $2005 b$ General expression of the gyroviscous force. Phys. Plasmas 12, 112301.

Ramos, J. J. 2008 Finite-Larmor-radius kinetic theory of a magnetized plasma in the macroscopic flow reference frame. Phys. Plasmas 15, 082106.

Riquelme, M. A., Quataert, E., Sharma, P. \& Spitkovsky, A. 2012 Local two-dimensional particle-in-cell simulations of the collisionless magnetorotational instability. Astrophys. J. 755, 50.

Roberts, K. V. \& TAYloR, J. B. 1962 Magnetohydrodynamic equations for finite Larmor radius. Phys. Rev. Lett. 8, 197-198.

Rosin, M. S., Schekochinin, A. A., Rincon, F. \& Cowley, S. C. 2011 A non-linear theory of the parallel firehose and gyrothermal instabilities in a weakly collisional plasma. Mon. Not. R. Astron. Soc. 413, 7-38. 
Rossi, C. 2015 Kelvin-Helmholtz instability at the magnetopause: theory and observations. PhD thesis, University of Pisa, Italy. (Available at https://etd.adm.unipi.it/t/etd-04132015-172025/).

Sarrat, M., Del Sarto, D. \& Ghizzo, A. 2016 Fluid description of Weibel-type instabilities via full pressure tensor dynamics. Europhys. Lett. 115, 45001.

Schekochinin, A. A., Cowley, S. C., Rincon, F. \& Rosin, M. S. 2010 Magnetofluid dynamics of magnetized cosmic plasma: firehose and gyrothermal instabilities. Mon. Not. R. Astron. Soc. 405, 291-300.

Schoeffler, K. M., Drake, J. F. \& Swisdak, M. 2011 The effects of plasma beta and anisotropy instabilities on the dynamics of reconnecting magnetic fields in the heliosheath. Astrophys. $J$. 743, 70 .

SCUdDER, J. \& DAUGhton, W. 2008 'Illuminating' electron diffusion regions of collisionless magnetic reconnection using electron agyrotropy. J. Geophys. Res. (Space Phys.) 113, A06222.

Servidio, S., Chasapis, A., Matthaeus, W. H., Perrone, D., Valentini, F., Parashar, T. N., Veltri, P., Gershman, D., Russell, C. T., Giles, B. et al. 2017 Magnetospheric multiscale observation of plasma velocity-space cascade: hermite representation and theory. Phys. Rev. Lett. 119 (20), 205101.

Servidio, S., Dmitruk, P., Greco, A., Wan, M., Donato, S., Cassak, P. A., Shay, M. A., Carbone, V. \& Matthaeus, W. H. 2011 Magnetic reconnection as an element of turbulence. Nonlinear Process. Geophys. 18, 675-695.

Servidio, S., Matthaeus, W. H., Shay, M. A., Dmitruk, P., Cassak, P. A. \& Wan, M. 2010 Statistics of magnetic reconnection in two-dimensional magnetohydrodynamic turbulence. Phys. Plasmas 17 (3), 032315.

Servidio, S., Osman, K. T., Valentini, F., Perrone, D., Califano, F., Chapman, S., Matthaeus, W. H. \& Veltri, P. 2014 Proton kinetic effects in vlasov and solar wind turbulence. Astrophys. J. Lett. 781, L27.

Servidio, S., Valentini, F., Califano, F. \& Veltri, P. 2012 Local kinetic effects in twodimensional plasma turbulence. Phys. Rev. Lett. 108 (4), 045001.

Servidio, S., Valentini, F., Perrone, D., Greco, A., Califano, F., Matthaeus, W. H. \& Veltri, P. 2015 A kinetic model of plasma turbulence. J. Plasma Phys. 81 (1), 325810107.

Shafranov, V. D. 1958 On magnetohydrodynamical equilibrium configurations. Sov. J. Expl Theor. Phys. 6, 545.

Siscoe, G. L., Crooker, N. U., Erickson, G. M., Sonnerup, B. U. Ö., Siebert, K. D., Weimer, D. R., White, W. W. \& MaYnard, N. C. 2000 Global Geometry of Magnetospheric Currents Inferred From MHD Simulations, Washington DC American Geophysical Union Geophysical Monograph Series, vol. 118, p. 41. American Geophysical Union, Washington, DC.

Sorathia, K. A., Merkin, V. G., Ukhorskiy, A. Y., Mauk, B. H. \& Sibeck, D. G. 2017 Energetic particle loss through the magnetopause: a combined global MHD and test-particle study. J. Geophys. Res. (Space Phys.) 122, 9329-9343.

Sorriso-Valvo, L., Perrone, D., Pezzi, O., Valentini, F., Servidio, S., Zouganelis, I. \& Veltri, P. 2018 Local energy transfer rate and kinetic processes: the fate of turbulent energy in two-dimensional Hybrid Vlasov-Maxwell numerical simulations. J. Plasma Phys. 84 (2), 725840201; 22 pp. ArXiv e-prints.

Squire, J., Kunz, M. W., Quataert, E. \& ScheKochinin, A. A. $2017 a$ Kinetic simulations of the interruption of large-amplitude shear-Alfvén waves in a high- $\beta$ plasma. Phys. Rev. Lett. 119 (15), 155101.

Squire, J., QuATAERT, E. \& KUnZ, M. W. 2017b Pressure-anisotropy-induced nonlinearities in the kinetic magnetorotational instability. J. Plasma Phys. 83 (6), 905830613.

Sulem, P. L. \& PASSOT, T. 2015 Landau fluid closures with nonlinear large-scale finite Larmor radius corrections for collisionless plasmas. J. Plasma Phys. 81, 325810103.

Sundberg, T., Boardsen, S. A., Slavin, J. A., Anderson, B. J., Korth, H., Zurbuchen, T. H., RAINes, J. M. \& Solomon, S. C. 2012 MESSENGER orbital observations of largeamplitude Kelvin-Helmholtz waves at Mercury's magnetopause. J. Geophys. Res. (Space Phys.) 117, A04216. 
TAYLOR, J. B. 1974 Relaxation of toroidal plasma and generation of reverse magnetic fields. Phys. Rev. Lett. 33, 1139.

Taylor, M. G. G. T., Hasegawa, H., Lavraud, B., Phan, T., Escoubet, C. P., Dunlop, M. W., Bogdanova, Y. V., Borg, A. L., Volwerk, M., Berchem, J. et al. 2012 Spatial distribution of rolled up Kelvin-Helmholtz vortices at Earth's dayside and flank magnetopause. Ann. Geophys. 30, 1025-1035.

Tenerani, A., Faganello, M., Califano, F. \& Pegoraro, F. 2011 Nonlinear vortex dynamics in an inhomogeneous magnetized plasma with a sheared velocity field. Plasma Phys. Control. Fusion 53 (1), 015003.

Tenerani, A., Velli, M. \& Hellinger, P. 2017 The parametric instability of Alfvén waves: effects of temperature anisotropy. Astrophys. J. 851, 99.

Terada, N., Machida, S. \& Shinagawa, H. 2002 Global hybrid simulation of the Kelvin-Helmholtz instability at the Venus ionopause. J. Geophys. Res. (Space Phys.) 107, 1471.

Valentini, F., Perrone, D., Stabile, S., Pezzi, O., Servidio, S., De Marco, R., Marcucci, F., Bruno, R., Lavraud, B., De Keyser, J. et al. 2016 Differential kinetic dynamics and heating of ions in the turbulent solar wind. New J. Phys. 18 (12), 125001.

Valentini, F., Servidio, S., Perrone, D., Califano, F., Matthaeus, W. H. \& Veltri, P. 2014 Hybrid Vlasov-Maxwell simulations of two-dimensional turbulence in plasmas. Phys. Plasmas 21 (8), 082307.

Valentini, F., Trávníček, P., Califano, F., Hellinger, P. \& Mangeney, A. 2007 A hybrid-Vlasov model based on the current advance method for the simulation of collisionless magnetized plasma. J. Comput. Phys. 225, 753-770.

Walsh, A. P., Hahland, S., Forsyth, C., Keesee, A. M., Kissinger, J., Li, K., Runov, A., SouceK, J., WALSh, B. M., Wing, S. et al. 2014 Dawn-dusk asymmetries in the coupled solar wind-magnetosphere-ionosphere system: a review. Ann. Geophys. 32, 705-737.

Wan, M., Matthaeus, W. H., Roytershteyn, V., Karimabadi, H., Parashar, T., Wu, P. \& SHAY, M. 2015 Intermittent dissipation and heating in 3D kinetic plasma turbulence. Phys. Rev. Lett. 114 (17), 175002.

YAJima, N. 1966 The effect of finite ion larmor radius on the propagation of magnetoacoustic waves. Prog. Theor. Phys. 36, 1-16.

Yang, Y., Matthaeus, W. H., Parashar, T. N., Haggerty, C. C., Roytershteyn, V., Daughton, W., Wan, M., Shi, Y. \& Chen, S. 2017 Energy transfer, pressure tensor, and heating of kinetic plasma. Phys. Plasmas 24 (7), 072306.

Yoon, P. H. 2017 Kinetic instabilities in the solar wind driven by temperature anisotropies. Rev. Mod. Plasma Phys. 1, 4. 